\title{
The effectiveness of marine protected areas for predator and prey with varying mobility
}

\author{
Sergei S. Pilyugin* \\ Jan Medlock ${ }^{\dagger}$ \\ Patrick De Leenheer $\ddagger$
}

April 8, 2016

\section{Keywords: Marine Protected Areas, predator prey, diffusion.}

\begin{abstract}
Marine protected areas (MPAs) are regions in the ocean where fishing is restricted or prohibited. Although several measures for MPA performance exist, here we focus on a specific one, namely the ratio of the steady state fish densities inside and outside the MPA. Several 2 patch models are proposed and analyzed mathematically. One patch represents the MPA, whereas the second patch represents the fishing ground. Fish move freely between both regions in a diffusive manner. Our main objective is to understand how fish mobility affects MPA performance. We show that MPA effectiveness decreases with fish mobility for single species models with logistic growth, and that densities inside and outside the MPA tend to equalize. This suggests that MPA performance is highest for the least mobile species. We then consider a 2 patch Lotka-Volterra predator-prey system. When one of the species moves, and the other does not, the ratio of the moving species first remains constant, and ultimately decreases with increased fish mobility, again with a tendency of equalization of the density in both regions. This suggests that MPA performance is not only highest for slow, but also for moderately mobile species. The discrepancy in MPA performance for single species models and for predator-prey models, confirms that MPA design requires an integrated, ecosystem-based approach. The mathematical approaches advocated here complement and enhance the numerical and theoretical approaches that are commonly applied to more complex models in the context of MPA design.
\end{abstract}

\section{Introduction}

Marine protected areas (MPAs) are regions in the ocean where fishing is restricted or prohibited. They have been used to manage fisheries for conservation and for habitat

*Department of Mathematics, University of Florida, email: pilyugin@ufl.edu. Supported in part by NSF DMS-1411853.

${ }^{\dagger}$ Department of Biomedical Sciences, Oregon State University, email: jan.medlock@oregonstate.edu

${ }^{\ddagger}$ Department of Mathematics, and Department of Integrative Biology, Oregon State University, email: deleenhp@math.oregonstate.edu. Supported in part by NSF DMS-1411853. 
restoration, while taking the economic interests of the fishing fleet into account, both at local as well as regional scales. For example, MPAs have been shown to increase the densities of harvested species inside of MPAs [16, 10], and MPAs may also increase fish densities outside of the MPA via spillover and/or larval export $[40,43,13]$. Spillover is the net movement of adult fish from the reserve into the fishing grounds. To increase yield from the fishery, the density of fish in the fishing grounds must increase enough to more than compensate for creation of the MPAs and the associated reduction in the area of the fishing grounds [40, 24, 1]. Spillover and larval export are essential to successfully increase fisheries yield, highlighting the importance of explicitly incorporating movement into models of MPA function and design [7, 36, 30]. Although some early mathematical models of MPAs did not explicitly include spatial movement $[34,18]$, there has also been an extensive numerical modeling literature where models do include fish mobility dating back to the work [2] by Beverton and Holt, see for instance [39] for single species models, and $[47,48,35,49,50,45]$ for numerical models that include predators and prey, or $[4,25,26,3]$ for numerical models with multiple trophic levels and structure.

A prevailing conclusion of these numerical investigations is that MPAs are believed to be effective for slow species, but far less so for more mobile species. This aligns with most field assessments of MPAs that show that densities of fish inside MPAs are much greater than densities outside of MPAs [16, 11, 10], but that increased species mobility diminishes this effect. In [36] it is shown that in a model with both larval and adult movement, species persistence may decrease sharply when the home range of the adults increases, and is less dependent on larval dispersal distances. This suggest that the adult dispersal rates and distances are important features in MPA design. However, recent studies have suggested that some of the putative beneficial effects of MPAs have alternative explanations, e.g. due to biases in siting of MPAs [37] or differential movement of fishes inside vs outside of MPAs $[12,30]$. In the recent review [5], it is shown that alternative hypotheses may lead to opposing effects of MPAs on disease prevalence, on density-dependent fish movement, and on the strength of trophic cascades within MPAs. On the other hand, the same review [5] indicates that MPAs tend to increase species diversity, and tend to decrease the variability of several measures, including fish biomass.

These findings indicate that there is currently not always a consensus about the factors that promote MPA effectiveness, and therefore a continued (re-)evaluation is necessary. We propose to contribute to this effort by using a more rigorous analytical approach, instead of the more traditional numerical and simulation-based methods. One of our findings is that MPAs can also be effective for species with intermediate movement rates, especially in the context of predator-prey systems. This agrees with the more recent empirical findings, and would enlarge the class of species that would benefit from protected areas.

The purpose of this paper in particular, is to mathematically investigate how differences in species impact MPA performance. We considered two kinds of variations in species ecology. First, species have variable mobility, which is represented by diffusivity parameters in our mathematical models. Second, species interact through a simple predator-prey relation, rather than considering isolated species.

Throughout the paper we will use a traditional measure of MPA effectiveness, namely the ratio of the densities of each species inside versus outside the MPA. 
Our analysis is guided by two specific questions which are part of the folklore in ecological modeling:

- Is increased mobility always stabilizing? That is, if species mobility is increased, will the system display a higher degree of stability, both locally as well as globally?

- Is increased mobility always equalizing? That is, if species mobility is increased, will the density ratio get closer to 1 ?

There are several other measures for MPA effectiveness that are commonly used in the theoretical and empirical literature, such as the fisheries yield, and the ratio of total biomass inside versus outside, or before versus after MPA implementation. For a similar mathematical analysis of the behavior of these other MPA measures with respect to fish mobility for a related single-species, spatially-explicit model, see [30]. As discussed in [51], the interpretation of the numerical values of all these MPA efficiency measures should always be considered in the appropriate context by MPA designers and managers. This is accomplished by incorporating as many features as possible about size and scale of the MPA, as well as the life-histories of the species that the MPA is intended to protect.

Empirical data [10] shows that the ratio of the density of fish inside versus outside an MPA increase as mobility of the fish increase. Specifically, among commercially exploited fishes, the least mobile species showed an approximately 2-fold increase and the most mobile species showed a 2.64-fold increase in abundance in response to protection. We first show that a simple model with only a single fish species contradicts this empirical result. However, as already mentioned, many fish species are part of a foodweb or of trophic cascades. Here we consider a predator-prey system with one predator and one prey. In some cases, this additional feature in the model does allow the ratios of both predator and prey to increase when the mobilities of either the predator or the prey is increased. For example, we will show that the prey steady state ratio increases with prey mobility, provided that the predator mobility is low.

\section{One-species MPA-FG model}

We considered an environment that consists of two patches. One is the MPA, and the other is the Fishing Ground (abbreviated to FG henceforth). The variable $u_{1}(t)$ is the fish density in the MPA at time $t$ and $u_{2}(t)$ is the fish density in the FG. Inside each patch, fish densities follow the logistic differential equation [27], with per-capita growth rates $r(1-u / K)$. In the $\mathrm{FG}$, there is removal of fish at the percapita harvesting rate $f$. Fish migrate between the two patches according to Fick's law: the migration rate is proportional to the difference of the fish densities in both patches, in the direction of the patch with lower density. These assumptions yield 
the system of equations:

$$
\begin{aligned}
\frac{\mathrm{d} u_{1}}{\mathrm{~d} t} & =r u_{1}\left(1-u_{1} / K\right)+D\left(u_{2}-u_{1}\right), \\
\frac{\mathrm{d} u_{2}}{\mathrm{~d} t} & =r u_{2}\left(1-u_{2} / K\right)-f u_{2}+D\left(u_{1}-u_{2}\right) \\
& =\tilde{r} u_{2}\left(1-u_{2} / \tilde{K}\right)+D\left(u_{1}-u_{2}\right),
\end{aligned}
$$

where

$$
\tilde{r}:=r-f \quad \text { and } \quad \tilde{K}:=K \frac{\tilde{r}}{r} \text {. }
$$

where the sign of $\tilde{r}$, or equivalently of $\tilde{K}$, may or may not be positive. We cannot directly solve for the non-extinction equilibrium of (1), but we can characterize this equilibrium. It satisfies

$$
\begin{aligned}
& u_{1}=\frac{K}{r}\left[r-D\left(1-\frac{u_{2}}{u_{1}}\right)\right]=\frac{K}{r}\left[r-D\left(1-\frac{1}{\rho}\right)\right], \\
& u_{2}=\frac{\tilde{K}}{\tilde{r}}\left[\tilde{r}+D\left(\frac{u_{1}}{u_{2}}-1\right)\right]=\frac{\tilde{K}}{\tilde{r}}[\tilde{r}+D(\rho-1)],
\end{aligned}
$$

where $\rho:=\frac{u_{1}}{u_{2}}$ is the ratio of the fish densities. Dividing the first by the second, noting that $\frac{K}{r}=\frac{\tilde{K}}{\tilde{r}}$, and rearranging gives the cubic equation for the equilibrium ratio

$$
F(\rho, D):=\rho^{3}+\left(\frac{\tilde{r}}{D}-1\right) \rho^{2}-\left(\frac{r}{D}-1\right) \rho-1=0 .
$$

For a fixed value of the diffusivity parameter $D$, any solution $\rho$ of (4) such that the corresponding values of $u_{1}$ and $u_{2}$ in (3) are positive, yields a positive steady state. It is shown in Theorem 1 in the Appendix that such a positive steady state is always unique, that it is globally stable, and that the corresponding ratio $\rho$ is always larger than 1. More precisely, if $f \leq 2 r$, then there is a positive steady state for every positive value of $D$. If $f>2 r$ on the other hand, a positive steady state exists only if $D<r \tilde{r} /(r+\tilde{r})=: D_{\max }$. Furthermore, it can be shown by implicit differentiation of (4) that the ratio $\rho$ is a decreasing function of $D$ in all cases. If $f \leq 2 r$, then $\rho \rightarrow 1$ as $D \rightarrow \infty$. But if $f>2 r$, then $\rho \rightarrow(f-r) / r>1$ as $D \rightarrow D_{\max }$. The expressions (3), and the fact that $\rho>1$ for all $D$, show that the steady state fish density in the MPA is always decreasing with $D$, whereas the steady state FG density is increasing. Hence, more mobile species will have higher FG densities, but lower MPA densities than less mobile species.

Note also that increasing diffusivity never destabilizes the unique positive steady state. Moreover, increased mobility is indeed equalizing because the steady-state ratio of MPA versus FG densities is always larger than 1, and decreases with higher mobility. These conclusions are similar to those in $[39,47,48]$, although those papers consider different models, different MPA measures, and use a numerical instead of analytical approach. In contrast, empirical data shows that this ratio increases [10]. 


\section{Predator-prey MPA-FG model}

The classic, appropriately scaled (see Appendix), Lotka-Volterra model of the population dynamics of predator and prey fish is

$$
\begin{aligned}
& \frac{\mathrm{d} x}{\mathrm{~d} t}=x(r-y), \\
& \frac{\mathrm{d} y}{\mathrm{~d} t}=y(x-d),
\end{aligned}
$$

where $x$ and $y$ are the densities of prey and predator fish, respectively $[32,46]$. The per capita net growth rate (equaling reproduction rate minus fishing rate) of the prey is $r$, and it is assumed to be positive, and the net death rate (equaling natural death rate plus fishing rate) of the predator is $d$, which is also assumed to be positive.

An interesting feature of this model concerns the values of the unique positive steady state $(\bar{x}, \bar{y})=(d, r)$. Indeed, the predator steady state value is completely determined by a prey characteristic, namely $r$, and similarly the prey steady state value is determined only by a predator feature, namely $d$.

Although model (5) provides an explanation for the oscillations in predator and prey densities, it has been criticized for several reasons. We mention two of these. First, in the absence of the predator, the prey would grow unbounded exponentially, which is clearly an unrealistic feature. Secondly, the system is structurally unstable. Indeed, arbitrarily small perturbations in the vector field can lead to drastically different dynamics, including blow-up of all solutions. These issues have been addressed in various ways. For example, the linear term $r x$ in the prey equation can be replaced by a logistic term $r x(1-x / K)$, and the mass-action term $x y$ can be replaced by a Holling type II function which takes the form

$$
\frac{m x}{x+a} y
$$

with positive parameters $m$ and $a$, resulting in the Rosenzweig-MacArthur model [42]. For small values of $K$, the Rosenzweig-MacArthur model has a unique, globally stable steady state, whereas for a certain value of $K$, the steady state undergoes a Hopf bifurcation, giving rise to a unique limit cycle. This phenomenon has been called the Paradox of Enrichment: A richer environment for the prey, encoded by a larger carrying capacity $K$, may destabilize the positive steady state. Further model modifications that avoid the Paradox of Enrichment are discussed in [28]. For more general predator-prey system modeling, see [6].

Despite all these attempts to build more realistic predator-prey models, we will use the basic Lotka-Volterra predator model (5) as the basic building block for the 2-patch model introduced below. One reason is that this basic form allows us to perform a more comprehensive global analysis of the 2-patch model. But the main reason is that our analytical work on more complicated 2-patch models with Rosenzweig-MacArthur interactions, did not behave fundamentally differently, and did not offer any new insights. Including this analysis could only obscure the true causes of the biological phenomena we wish to understand, and hence we have decided not to include it here. Nevertheless, some numerical evidence illustrating our findings will be presented in the Discussion Section. 


\section{Coupled MPA-FG predator prey model}

We extend the Lotka-Volterra model (5) to a 2-patch MPA-FG system. We assume Lotka-Volterra interactions within each patch, and include constant per capita fishing rates $f_{x}$ and $f_{y}$ for prey and predator respectively in the FG. Coupling between MPA and FG occurs via diffusive coupling terms. The model equations are:

$$
\begin{aligned}
& \frac{\mathrm{d} x_{1}}{\mathrm{~d} t}=x_{1}\left(r-y_{1}\right)+D_{x}\left(x_{2}-x_{1}\right), \\
& \frac{\mathrm{d} y_{1}}{\mathrm{~d} t}=y_{1}\left(x_{1}-d\right)+D_{y}\left(y_{2}-y_{1}\right), \\
& \frac{\mathrm{d} x_{2}}{\mathrm{~d} t}=x_{2}\left(\tilde{r}-y_{2}\right)+D_{x}\left(x_{1}-x_{2}\right), \\
& \frac{\mathrm{d} y_{2}}{\mathrm{~d} t}=y_{2}\left(x_{2}-\tilde{d}\right)+D_{y}\left(y_{1}-y_{2}\right),
\end{aligned}
$$

where

$$
\tilde{r}:=r-f_{x}<r, \quad \tilde{d}:=d+f_{y}>d,
$$

and $D_{x}$ and $D_{y}$ are the diffusivities of prey and predator. We assume henceforth that:

$$
\tilde{r}>0,
$$

i.e. we assume that prey fishing occurs at moderate levels. First, when there is no diffusive coupling between the patches (i.e. when $D_{x}=D_{y}=0$ ), the non-extinction steady-state equals $(d, r, \tilde{d}, \tilde{r})$. The corresponding steady state ratio of the prey densities inside versus outside the MPA is

$$
\rho_{x}^{0}:=\frac{x_{1}^{*}}{x_{2}^{*}}=\frac{d}{\tilde{d}}<1 .
$$

Notice that fishing of the predator causes the prey density to be larger in the FG than in the MPA. This constitutes a first remarkable difference with what happens in the single-species case described by a logistic equation, where this steady-state ratio is larger than 1. For the predator, there is an opposite effect. Indeed, the predator steady state ratio is

$$
\rho_{y}^{0}:=\frac{y_{1}^{*}}{y_{2}^{*}}=\frac{r}{\tilde{r}}>1,
$$

whenever prey is being fished. Thus, the predator density in the MPA is larger than in the FG.

Our goal is to understand the dynamics when predator and/or prey are moving between MPA and FG. The most complete picture of the system's behavior arises when one of the species moves, but the other does not. We address these two cases first, before continuing on to the general case where both species move.

Predators move, but prey do not: $D_{x}=0$ and $D_{y}>0$. It is shown in Theorem 2 in the Appendix that the non-extinction steady state is

$$
E_{y}^{*}=\left(x_{1}^{*}, y_{1}^{*}, x_{2}^{*}, y_{2}^{*}\right)=\left(d+D_{y}\left(1-\left(\rho_{y}^{0}\right)^{-1}\right), r, \tilde{d}+D_{y}\left(1-\rho_{y}^{0}\right), \tilde{r}\right),
$$


where $\rho_{y}^{0}>1$ was defined in $(10) . E_{y}^{*}$ is the unique positive steady state if and only if

$$
0 \leq D_{y}<\frac{\tilde{r} \tilde{d}}{r-\tilde{r}}
$$

Note that the predator steady-state values $r$ and $\tilde{r}$ in MPA and FG respectively, are constant, and do not depend on the predator mobility $D_{y}$. This is because the steady state values of the predator, are determined by the prey equations, and these equations are not affected by the predator diffusivity $D_{y}$. Since the the steady-state densities for the predator don't depend on $D_{y}$, neither does their ratio. In contrast, the prey steady-state values do depend on $D_{y}$. The MPA prey density increases with $D_{y}$, whereas the FG density decreases to zero. Hence, the prey ratio increases with $D_{y}$. It crosses the value 1 at $D_{y}^{\text {crit }}=(\tilde{d}-d) /\left(\rho_{y}^{0}-\left(\rho_{y}^{0}\right)^{-1}\right)$, and it approaches infinity when $D_{y}$ approaches $\tilde{r} \tilde{d} /(r-\tilde{r})$, see Figure 1 .

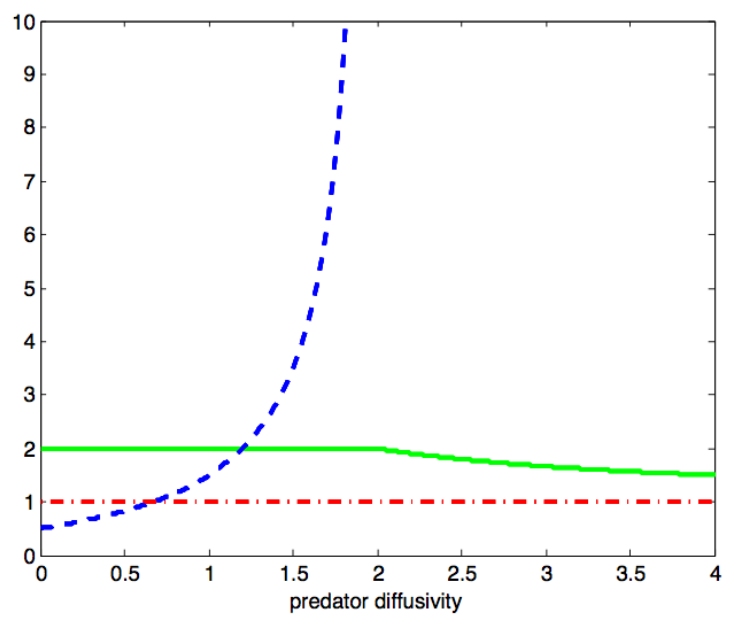

Figure 1: Steady state MPA/FG ratios when the predator moves, but the prey does not, versus predator diffusivity $D_{y}$ (Prey ratio in blue dashed line, predator ratio in solid green). The prey ratio crosses 1 when $D_{y}=D_{y}^{\text {crit }}=2 / 3$ per time unit, showing that increased predator diffusivity is not always equalizing. Parameters used: $r=2$, $f_{x}=1, d=1, f_{y}=1$, all in units of $1 /$ time. The positive steady state $E_{y}^{*}$ exists and is globally stable for $0 \leq D_{y}<2$. The boundary steady state $E_{\mathrm{MPA}}^{*}$ is globally stable for $D_{y}>2$.

In Appendix C.5 we show that there are other steady states on the boundary. One of these is

$$
E_{\mathrm{MPA}}^{*}=\left(\frac{\left(d+D_{y}\right)\left(\tilde{d}+D_{y}\right)-D_{y}^{2}}{\tilde{d}+D_{y}}, r, 0, \frac{r D_{y}}{\tilde{d}+D_{y}}\right),
$$

and it exists for all positive values of $D_{y}$. When $D_{y}$ is in the range of (12), then $E_{\mathrm{MPA}}^{*}$ is unstable, but when

$$
D_{y}>\frac{\tilde{r} \tilde{d}}{r-\tilde{r}}
$$

then $E_{\mathrm{MPA}}^{*}$ is stable and attracts all positive solutions. This suggests that a transcritical bifurcation occurs at $D_{y}=\tilde{r} \tilde{d} /(r-\tilde{r})$. The positive steady state $E_{y}^{*}$ ceases 
to exist when $D_{y}$ is pushed beyond this bifurcation value, and it exchanges stability with the boundary steady state $E_{\mathrm{MPA}}^{*}$.

Let us offer a biological explanation of these results. Assume that we start with a configuration where not only the prey, but also the predator doesn't move, i.e. $D_{y}=0$. Then the MPA density of the predator equals $r$, which is larger than the FG density, where it equals $\tilde{r}$. Now suppose that $D_{y}$ is increased slightly. A key fact is that this increase in $D_{y}$ does not change the predator steady-state densities in MPA or FG. These remain equal to $r$ and $\tilde{r}$ respectively. This follows immediately from the prey steady state equations $(7 a)$ and $(7 c)$ because the prey does not move $\left(D_{x}=0\right)$, and because there is no density dependence in the prey growth. As soon as $D_{y}$ becomes positive, there will be a net flux of predators from MPA to FG. This is a consequence of Fick's law which stipulates that movement is directed from locations of high towards low density. But this net flux of predators will decrease the predator density in the MPA (and increases it in the FG). The reduction of predators in the MPA will benefit prey there, but similarly, the increase in predators in the FG is harmful for prey there. Thus, MPA prey density will increase, but FG prey density will decrease, and consequently, the ratio of MPA/FG prey densities must increase.

What is truly remarkable, is that the same argument remains valid when we increase the predator diffusivity starting from any positive value, and not just starting from zero. Indeed, as already mentioned, the increase of $D_{y}$ does not affect the values of the predator steady state values in MPA and FG. As a result the net flux caused by an increased predator diffusivity, is always directed from the MPA to the FG. But then just as before, the prey density will increase in the MPA (but decrease in the FG), and thus the ratio of the MPA/FG prey densities must increase. Of course, this is sustainable only as long as the (decreasing) prey density in the FG remains positive, and this only happens for a finite range of predator diffusivities, as indicated by the range in (12). We have shown that when $D_{y}=0$, the prey ratio is less than one, and when $D_{y}$ approaches its upper bound, the ratio grows unbounded (because the denominator of the ratio goes to zero, whereas its numerator remains bounded). Since the ratio always increases with $D_{y}$, there must be a unique value of $D_{y}$ where the prey ratio equals one. The relevance of this threshold is that it accurately determines when increasing predator diffusivity has an equalizing effect on prey densities: Equalization occurs as long as the predator diffusivity remains below the threshold. For values of $D_{y}$ outside the range of (12), there is no longer a positive steady state: The predator diffusivity has become too large, and instead a boundary steady state for which there is no more prey in the FG is now globally stable. In other words, the predator's diffusivity has driven the prey to extinction in the FG. Remarkably, the prey density in the MPA is increasing with respect to predator diffusivity, and so is the predator density in the FG.

Prey move, but predators do not: $D_{x}>0$ and $D_{y}=0$. In the case where only the prey moves, there are, depending on the value of the prey diffusivity $D_{x}$ relative to 3 threshold values, distinct possible dynamics. These thresholds for $D_{x}$ are $\tilde{r}, r$ and $\bar{D}_{x}=\tilde{r} \tilde{d} /(\tilde{d}-d)$. Since $\tilde{r}<r$ and $\tilde{d}>d$, it follows that $\tilde{r}<\bar{D}_{x}$. Let us assume that $\tilde{r}<\bar{D}_{x}<r$ (the case $\tilde{r}<r<\bar{D}_{x}$ can be handled in a similar way). We now let $D_{x}$ vary from 0 to $\infty$. First, as long as $D_{x}<\bar{D}_{x}$, it is shown in Theorem 3 
in the Appendix, that there is a unique positive steady state

$$
E_{x}^{*}=\left(d, r+D_{x}(\tilde{d}-d) / d, \tilde{d}, \tilde{r}-D_{x}(\tilde{d}-d) / \tilde{d}\right)
$$

and it is globally stable with respect to positive solutions. At this steady state, both species coexist in both regions. The steady-state densities of the prey do not depend on $D_{x}$, and therefore neither does the prey's steady state ratio. Once again, this is due to the fact that the predator does not move. In contrast, the predator steady state density is increasing in the MPA, but decreasing in the FG when $D_{x}$ increases; consequently the predator's steady-state ratio is increasing. This ratio is always larger than 1 , and approaches infinity as $D_{x}$ approaches $\bar{D}_{x}$, see Figure 2 . In Theorem 6 in the Appendix, we show that when $D_{x}$ increases from zero beyond the first threshold $\tilde{r}$, an (unstable) boundary steady state $E_{\text {MPA }}^{* *}$ shows up where the predator exists only in the MPA:

$$
E_{\mathrm{MPA}}^{* *}=\left(d, r+\tilde{r} D_{x} /\left(D_{x}-\tilde{r}\right), d D_{x} /\left(D_{x}-\tilde{r}\right), 0\right),
$$

When $D_{x}$ is further increased beyond $\bar{D}_{x}$, there is a transcritical bifurcation, where the positive steady state $E_{x}^{*}$ disappears via the boundary steady state $E_{\text {MPA }}^{* *}$, which in turn becomes globally stable. The predator can no longer survive in the fishing ground when $D_{x}$ surpasses $\bar{D}_{x}$. The prey's steady state ratio at $E_{\text {MPA }}^{* *}$ is no longer constant, but increases with $D_{x}$, and approaches 1 as $D_{x}$ approaches infinity. Finally, when $D_{x}$ is pushed above $r$, a new steady state $E_{\mathrm{FG}}^{* *}$ shows up, where the predator can only exist in the fishing ground, but this steady state is always unstable. In summary, for slow prey, the prey's steady state densities in MPA and FG are constant, and always higher in the FG. This causes a constant flux of prey that move from FG into the MPA, and as long as there is some surplus prey in the FG, the predator can survive there. But when the prey moves too fast, there is no surplus prey left in the FG and the predator collapses there. The prey ratio is now no longer constant, but increases with prey mobility. Furthermore, in this regime, increased prey mobility has an equalizing effect on prey densities in MPA and FG.

Both predators and prey move: $D_{x}>0$ and $D_{y}>0$. In Theorem 4 in the Appendix, we show that for any positive pair of $D_{x}$ and $D_{y}$ diffusivities, there is a unique positive steady state. The predator steady-state ratio $\rho_{y}$ is always larger than 1 , and the prey steady-state ratio $\rho_{x}$ is less than 1 (or larger than 1 ) if $D_{y}$ is less than $D_{y}^{\text {crit }}\left(\right.$ respectively, larger than $\left.D_{y}^{\text {crit }}\right)$, where $D_{y}^{\text {crit }}:=(d-\tilde{d}) /\left(\rho_{y}^{0}-\left(\rho_{y}^{0}\right)^{-1}\right)$ :

$$
\rho_{y}>1 \text { and } \rho_{x}\left\{\begin{array}{l}
<1 \text { if } D_{y}<D_{y}^{\text {crit }} \\
>1 \text { if } D_{y}>D_{y}^{\text {crit }}
\end{array}\right.
$$

In Theorem 5 we show that if $D_{y}<D_{y}^{\text {crit }}$, then the following sensitivities hold:

$$
\frac{\partial \rho_{x}}{\partial D_{x}}>0, \quad \frac{\partial \rho_{y}}{\partial D_{x}}>0, \quad \frac{\partial \rho_{x}}{\partial D_{y}}>0, \quad \frac{\partial \rho_{y}}{\partial D_{y}}<0 .
$$

and that the positive steady state is locally stable.

On the other hand, if $D_{y}>D_{y}^{c r i t}$, then the following sensitivities hold:

$$
\frac{\partial \rho_{x}}{\partial D_{x}}<0, \quad \frac{\partial \rho_{y}}{\partial D_{x}}<0, \quad \frac{\partial \rho_{x}}{\partial D_{y}}>0, \quad \frac{\partial \rho_{y}}{\partial D_{y}}<0 .
$$




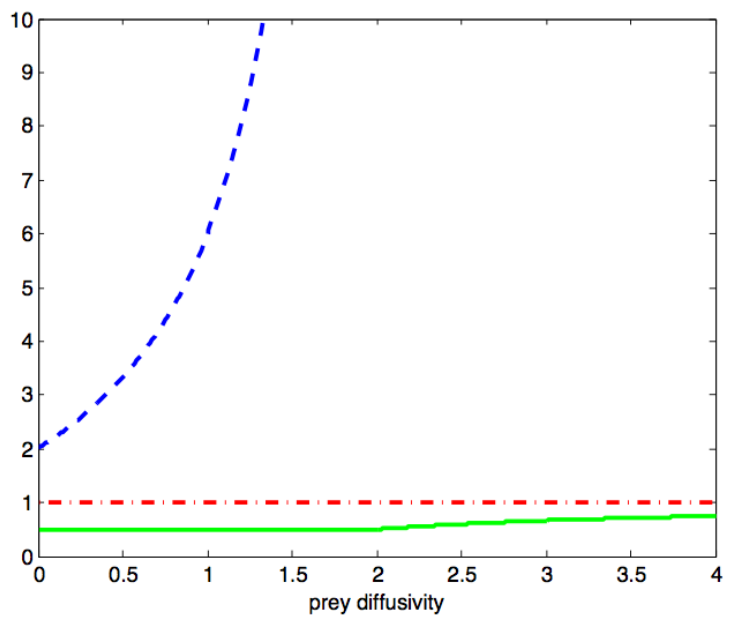

Figure 2: Steady state MPA/FG ratios when the prey moves, but the predator does not, versus prey diffusivity $D_{x}$ (Predator ratio in blue dashed line, prey ratio in solid green). The predator ratio is always above 1 , and it increases with $D_{x}$, showing that increased prey diffusivity is never equalizing the MPA and FG steady state densities. Parameters used: $r=2, f_{x}=1, d=1, f_{y}=1$, all in units of $1 /$ time. The positive steady state $E_{x}^{*}$ exists and is globally stable for $0 \leq D_{x}<2$. The boundary steady state $E_{\mathrm{MPA}}^{* *}$ is globally stable for $D_{x} \geq 2$.

In this case, we currently do not have a proof of local stability of the positive steady state.

We can summarize some of the conclusions encoded by (15), (16) and (17) as follows:

Increased diffusivity of one of the species, always leads to an equalizing effect on that same species.

Let us prove this statement for the prey species for example (the proof is similar for the predator). We distinguish two cases, depending on whether the predator diffusivity $D_{y}$ is above or below the threshold $D_{y}^{\text {crit }}$. If $D_{y}<D_{y}^{\text {crit }}$, then $\rho_{x}<1$, and according to the first sensitivity inequality in (16), $\rho_{x}$ increases with $D_{x}$, establishing the claim above. Similarly, if $D_{y}>D_{y}^{c r i t}$, then $\rho_{x}>1$, and according to the first sensitivity inequality in (17), $\rho_{x}$ now decreases with $D_{x}$, once again establishing the claim.

The effect of an increase of the mobility of one species on the other species is more complicated. As long as $D_{y}$ is small (i.e. $D_{y}<D_{y}^{\text {crit }}$ ), then (16) predicts that an increased prey mobility prevents the predator ratios from equalizing, whereas increased predator mobility promotes equalization of the prey densities. For large values of $D_{y}$ (i.e. $D_{y}>D_{y}^{\text {crit }}$ ) however, these conclusions are reversed, according to (17).

\section{Discussion}

From single species models to predator-prey systems. Single-species models that include movement are frequently represented as reaction-diffusion equations, see 
[9]. In an MPA context such PDE models have been used to investigate the effects of a higher diffusion coefficient on various measures of MPA effectiveness, both through simulations [38] and elementary mathematical analysis [30]. The results of these papers are consistent with those presented in Theorem 1, although the model considered here is a 2-patch model in which space is discrete and consists of two points, rather than a continuum as in the aforementioned PDEs. The model investigated here also ignores size structure (and hence also possibly size-dependent fecundities). Whereas all these single species models, including the one investigated here, predict that higher diffusivity gives rise to a lower density ratio, this conclusion contradicts the empirical data in [10]. This paradox motivated us to the shift our focus from single species models to two species models, and in particular to predatorprey systems.

Of course, this specific choice, is only one of many, and several others have already been proposed elsewhere. For example, in [30], a single species PDE model was analyzed that includes a specific movement bias at the boundary between the MPA and FG. The bias was assumed to be directed towards the MPA because the MPA affords extra protection from harvesting. It was shown that for sufficiently high bias parameters the density ratio is increasing with diffusivity.

In this paper, we explored a 2 patch predator-prey model. When only one of the species moves, the steady state ratio of the non-moving species, increases with the diffusivity parameter of the moving species. The steady state ratio of the moving species always tends to one with increased diffusivity, which shows that increased diffusivity has an equalizing effect on MPA and FG densities. If this moving species is a prey, then the prey ratio is increasing, just like the data in [10] (if it is a predator, the ratio is still decreasing, like in the single species model).

In the general case where both predator and prey move, we showed that increased diffusivity of one species, always has an equalizing effect on that same species. For a predator, the ratio is always decreasing. For a prey, it depends: When the predator is slow, the prey ratio is decreasing, but when the predator is fast, the prey ratio is increasing with prey diffusivity. The latter result can therefore be used to reconcile theoretical model predictions with the empirical data in [10]. For instance, if many of the species in the study of [10] are prey species in a predator-prey system with sufficiently slow predator, then there is agreement between model and data. But if they are mostly predators, this would not be the case, and other models used to explain the discrepancy.

Reality is of course not that simple; many species are part of larger networks, and may be both predator as well as prey. Not only predator-prey interactions are relevant to MPA assessment and design. For example, most fish populations exhibit stage structure with larval, juvenile and adult stages, or more complicated trophic structures $[41,26]$. None of our models include more than 2 trophic levels. Nevertheless, nothing precludes the use of the mathematical methods exploited here, in the analysis of more complicated models.

From synchronization to global stability. There is a rich literature on the diffusive coupling of 2 or more systems described by nonlinear ODEs, see $[17,19,15,20,29,23]$. In [17] for instance, it is shown that certain diffusively coupled Lotka-Volterra models (not necessarily of the predator-prey type) may under certain conditions, be globally stable. However, a crucial assumption in that 
work is that the dynamics in each patch is the same. Clearly, this assumption is violated in an MPA-FG context. From a wider perspective, the work by Hale in [15] is focused on the diffusive coupling of rather arbitrary nonlinear ODE models. When the dynamics of the uncoupled models is the same, it is shown that synchronization occurs. That is, the 'diagonal' of the state space attracts the system's solutions for sufficiently large diffusivity parameters. In other words, the state of all the patches converges to the same-yet, not necessarily constant- value, provided that the diffusivity parameter is large enough. Although these results are related to ours, there are three fundamental differences. First, synchronization does not imply that the coupled system has a globally stable steady state. Indeed, one can for example synchronize two chaotic Lorenz attractors through diffusive coupling. Secondly, synchronization only occurs under the assumption that the uncoupled systems have the same dynamics, which is obviously not the case in an MPA context. [If the dynamics of the coupled systems are sufficiently close to each other, then a weaker phenomenon, namely that of almost synchronization, may occur. A system almost synchronizes when its state merely converges to arbitrarily small neighborhoods of the diagonal for all sufficiently strong coupling terms]. Thirdly, synchronization, as well as almost synchronization, requires that the diffusivity parameter is sufficiently large. Our focus here is on (i) cases where the uncoupled systems differ significantly (MPA versus FG dynamics) (ii) a more detailed understanding of how they behave asymptotically when coupled diffusively; in particular on global stability (iii) scenarios in which the diffusivities can be small, and even zero. More recent related work in the context of synchronization of many coupled predator-prey systems has appeared in [19]. There, the predator and prey interact according to the Rosenzweig-MacArthur model, and the emphasis is on how the network structure affects the synchronization of clusters in the resulting coupled system.

Let us now return to some of the fundamental ecological questions underlying this work:

1. Is diffusion stabilizing? The smoothing effects of diffusion are well-known, but when coupled with reaction terms that model various biological and physical processes (growth, decay, consumption, (bio)chemical reactions etc), the combined effect of diffusion and reactions may in fact destabilize a system, as shown by Alan Turing. Turing considered a system of 2 reaction-diffusion equations with homogeneous reaction terms, and showed that the homogeneous steady state corresponding to a steady state of the reaction terms alone, may become unstable when diffusion of both species is allowed. However, Turing also identified which type of reaction systems have the potential to achieve this (in addition to pointing out that the diffusivities of both species should be different), and he showed that predator-prey interactions are not among these. Similar results can be obtained in diffusively coupled patch models ${ }^{1}$, and although the predator-prey dynamics is not homogeneous across the patches in our models, our main results are consistent with Turing's observations, that diffusion cannot destabilize our coupled predator-prey systems. Specifically, when only one of the species moves, we have shown that the coupled

\footnotetext{
${ }^{1}$ Interestingly, for diffusively coupled predator-prey systems with a so-called quiescence phase considered in [14], diffusive instability is possible. But such systems are characterized by an inherent heterogeneity in the reaction dynamics in the patches (any reaction dynamics is shut off in the quiescent phase), whereas these dynamics are assumed to be the same in Turing's context.
} 
MPA-FG system exhibits a unique positive and globally stable steady state, at least for some range of the diffusivity parameter of the moving species (Theorems 2 and 3). When both species move, there is still a unique positive steady state, and we have shown that it is locally stable if the predator diffusivity is sufficiently small (Theorem 2).

On the other hand, there is an interesting phenomenon that can occur here. Prior to coupling (i.e. when both predator and prey diffusivities are zero), the uncoupled MPA-FG system has a neutrally stable steady state, because the linearization has 4 eigenvalues on the imaginary axis. When coupling occurs by making at least one of the diffusivities positive, the steady state of the coupled MPA-FG system is asymptotically stable: all 4 eigenvalues of the linearization are to the left of the imaginary axis. But further increasing the diffusivity parameter does not necessarily push these eigenvalues more to the left. In fact, Theorem 2 (as well as a similar result in Theorem 3) revealed that if $\tilde{r} \tilde{d}>r d$, then the unique positive steady state will once again be neutrally stable when $D_{y}=D_{y}^{*}$. This means that, whereas initially, larger diffusivity parameters tend to stabilize the steady state, even larger values of this parameter will bring the steady state closer to the region of instability again. In other words, a larger diffusivity parameter does not necessarily imply a more stable steady state.

In [21] the effect of a different kind of coupling in a 2 patch predator-prey system is established. Rather than assuming that the rate of movement of predator and prey is governed by the imbalance between the densities of each species in the 2 patches, it is assumed that the flux of the prey depends on the density of the predator, and vice versa. The underlying idea of this specific flux is that prey will tend to flee the patch where the predator is at high density, whereas predators prefer to move to the patch where the prey density is high. Interestingly, although the coupling terms proposed here are different from those in [21], the conclusions for the models are the same: In both cases, coupling cannot destabilize the system. In the related work [22] by the same authors, a detailed bifurcation analysis is performed for a coupled Rosenzweig-MacArthur model with two patches in which only the predator moves. Predator movement is based on prey densities, and parametrized by a parameter that quantifies how mobile a predator is while it is handling its prey. The focus of [22] is on whether or not this kind of predator mobility can always dampen the severe oscillations which are prevalent in predator-prey systems that lack a mobility component, and it is shown that this is not the case- see also [33] for more recent work on a PDE model. The contrast between the stabilizing [21] versus the nonstabilizing [22] effect due to increased mobility, demonstrates how important it is to understand what exactly the driving forces are behind species mobility.

The work of [29] is closely related to ours and also establishes the global stability of a positive steady state in a model of a prey which moves between 2 patches, and a predator who does not perceive these patches, and whose density is therefore modeled by a single variable. The predator-prey interactions are allowed to be more general than the Lotka-Volterra type, yet the coupled system has only 3 instead of 4 state variables. Also closely related is the work of [31], which was in part inspired by some fascinating simulations in [23], used to explain the Paradox of Enrichment by allowing the coupling of predator-prey systems of the Rosenzweig-MacArthur type. Although the models in [31] assume Rosenzweig-MacArthur predator-prey 
interactions, the results are weaker since global stability is only proved when the patch dynamics are the same, whereas here we prove global stability in the case of unequal patch dynamics, provided that at least one of the predator or prey species does not move.

To test how general our conclusions are, we explored more realistic, diffusively coupled 2 patch predator-prey system using Rosenweig-MacArthur predator-prey dynamics instead of the simple Lotka-Volterra analyzed in this paper. Although the mathematical analysis is more complicated than in the Lotka-Volterra case, these more realistic models behave similarly as the ones considered in this paper. Since adding additional complicating features to models that do not fundamentally change their underlying behavior, only has an obscuring effect -because it does not allow us to disentangle which feature is responsible for what effect- we have chosen not to include these analytical results for the more general models. Nevertheless, we present some numerical results to illustrate our findings, for the following model:

$$
\begin{aligned}
& \dot{x}_{1}=r x_{1}\left(1-\frac{x_{1}}{K}\right)-\frac{m x_{1}}{a+x_{1}} y_{1}+D_{x}\left(x_{2}-x_{1}\right) \\
& \dot{y}_{1}=y_{1}\left(\frac{m x_{1}}{a+x_{1}}-d\right) \\
& \dot{x}_{2}=\tilde{r} x_{2}\left(1-\frac{x_{2}}{\tilde{K}}\right)-\frac{m x_{2}}{a+x_{2}} y_{2}+D_{x}\left(x_{1}-x_{2}\right) \\
& \dot{y}_{2}=y_{2}\left(\frac{m x_{2}}{a+x_{2}}-\tilde{d}\right)
\end{aligned}
$$

Here, only the prey moves, and $\tilde{r}=r-f_{x}, \tilde{K}=K \tilde{r} / r$, and $\tilde{d}=d+f_{y}$. In Figure 3 we plot the steady state ratios of the positive steady state (for those diffusivity values $D_{x}$ for which it exists), as well as the ratios corresponding to a boundary steady state corresponding to a case where there is no more prey left in the FG.

We see that qualitatively, these steady state ratios behave like the corresponding ratios in case of Lotka-Volterra interactions, see Figure 2. Thus, despite the simplicity of the Lotka-Volterra models, they seem to be able to capture well how MPA effectiveness changes with species mobility.

2. Is diffusion equalizing? First of all, it is natural to inquire whether or not increased diffusivity of one species has an equalizing effect on the steady state ratio of that same species. When only one of the species moves -be it the predator or the prey- the answer is always no. This is because the steady state densities of the diffusing species are independent of the diffusivity parameter. Consequently, the steady state ratio remains constant for that species, and thus there is no effect whatsoever. In contrast, when both the predator and the prey move, the answer is always yes according to Theorem 5 .

Secondly, one may inquire about the effect of an increased diffusivity of one species, on the steady state ratio of the other species. According to Theorem 2, when the predator moves, but the prey doesn't, the prey densities are equalizing as long as the predator diffusivity is not too large. Beyond a critical threshold however, the prey ratio is larger than one and continues to grows with increased predator diffusivity. When the prey moves and the predator doesn't, Theorem 3 shows that increased prey diffusivity is never equalizing for the predator. And when 


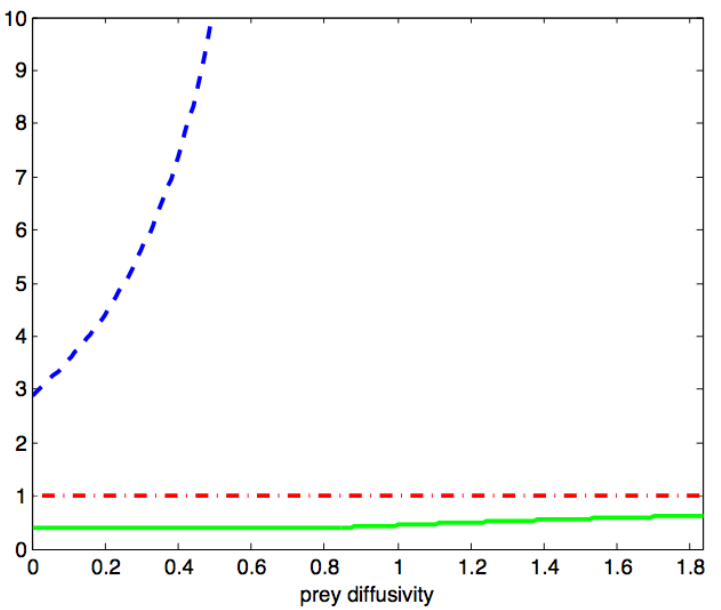

Figure 3: Steady state MPA/FG ratios in a Rosenzweig-MacArthur coupled patch model when the prey moves, but the predator does not, versus prey diffusivity $D_{x}$ (Predator ratio in blue dashed line, prey ratio in solid green). The predator ratio is always above 1 , and it increases with $D_{x}$, showing that increased prey diffusivity is never equalizing the predator densities. Parameters used: $r=2, f_{x}=1, K=2$, $m=3, a=1 d=0.5, f_{y}=0.5$. The positive steady state $E_{x}^{*}$ exists for $0 \leq D_{x}<$ $5 / 6$, and the boundary steady state $E_{\mathrm{MPA}}^{* *}$ for $D_{x} \geq 5 / 6$.

both species move, Theorem 5 shows that there are exactly 2 situations in which increased diffusivity is not equalizing. It occurs for the predator with respect to an increasing prey diffusivity, provided that the predator is slow. And it also happens for the prey with respect to an increasing predator diffusivity, when the predator is fast.

Some remarks for policy makers Policy makers face the important decision on when and where to implement MPAs. On the one hand, they need to protect fish from over-harvesting and possible collapse. And on the other hand, they should weigh this against the economic interests of fishermen. Difficult as such decisions may seem to be, this paper has shown that other factors can complicate matters even more. The focus here has been on two specific features:

1. How species mobility affects various measures of MPA effectiveness?

2. How important is it to take into account whether a given species is part of a predator-prey system?

We have shown that in case of a single adult species, the MPA/FG ratio is always larger than one, but decreases for more mobile fish. This is one reason why MPAs are often believed to be more effective for less mobile species.

However, suppose that this same species is known to be the predator in a predator-prey system in which the prey does not move. Then the MPA/FG ratio of this predator is still always larger than one, and it also ultimately decreases, but only after remaining constant for a large interval of predator mobilities. In other words, in this case, MPAs are predicted to be effective for many species, with mobilities ranging from low to moderately high. Thus, MPAs might be an appro- 
priate management tool for a larger class of species than anticipated on the basis of predictions of single species models. 


\section{A Appendix: One-species MPA-FG model}

Theorem 1. When system (1) admits a positive steady state $\left(u_{1}^{*}, u_{2}^{*}\right)$, it is unique, stable and attracts all positive solutions. The ratio $\rho^{*}=u_{1}^{*} / u_{2}^{*}>1$ is a decreasing function of the diffusivity $D$.

- If $f \leq 2 r$, the positive steady state exists for all values $D>0$. The ratio $\rho^{*}$ converges to 1 as $D \rightarrow \infty$.

- If $f>2 r$, the positive steady state exists only if $0<D<D_{\max }:=\frac{r \tilde{r}}{r+\tilde{r}}$. The ratio $\rho^{*}$ converges to $\frac{f-r}{r}$ as $D \rightarrow D_{\max }$.

Proof. Recall that positive steady states satisfy (3), where $\rho:=\frac{u_{1}}{u_{2}}$ is the ratio of the fish densities, and that therefore $\rho$ satisfies (4). Whenever convenient, we will also use an equivalent expression for $F$ :

$$
F(\rho, D)=(\rho-1)\left(\rho^{2}+1\right)+\frac{\tilde{r}}{D} \rho^{2}-\frac{r}{D} \rho
$$

We begin by noting that the root of interest must be greater than $\max \left(1,1-\frac{\tilde{r}}{D}\right)$. Indeed, if $0<\tilde{r}<r$, then for all $0<\rho \leq 1$, we have

$$
F(\rho, D)<(\rho-1)\left(\rho^{2}+1\right)+\frac{r}{D} \rho^{2}-\frac{r}{D} \rho=(\rho-1)\left(\rho^{2}+\frac{r}{D} \rho+1\right)<0,
$$

hence $F$ has no roots in the interval $(0,1]$ and $1-\tilde{r} / D<1$. If, on the other hand, $\tilde{r}<0<r$, then the root $\rho^{*}$ corresponding to a positive equilibrium must satisfy $\rho^{*}>1-\tilde{r} / D>1$ to ensure that $u_{F G}>0$, see (3). Now we will argue that equation (4) admits at most one root $\rho^{*}>\max \left(1,1-\frac{\tilde{r}}{D}\right)$.

Consider any value $\rho^{*}$ such that $F\left(\rho^{*}, D\right)=0$ and $\rho^{*}>\max (1,1-\tilde{r} / D)$. Differentiating $F$ with respect to $\rho$, we obtain

$$
F_{\rho}(\rho, D)=\left(3 \rho^{2}-2 \rho+1\right)+\frac{2 \tilde{r} \rho-r}{D}=\left(3 \rho^{2}-2 \rho+1\right)+\frac{\tilde{r} \rho-r}{D}+\frac{\tilde{r} \rho}{D}
$$

Since

$$
\frac{\tilde{r} \rho^{*}-r}{D}=-\left(\rho^{*}\right)^{2}+\rho^{*}-1+\left(\rho^{*}\right)^{-1}
$$

we find that

$$
F_{\rho}\left(\rho^{*}, D\right)=2\left(\rho^{*}\right)^{2}-\rho^{*}+\left(\rho^{*}\right)^{-1}+\frac{\tilde{r} \rho^{*}}{D}=\rho^{*}\left(\rho^{*}-(1-\tilde{r} / D)\right)+\left(\rho^{*}\right)^{2}+\left(\rho^{*}\right)^{-1},
$$

which is positive since $\rho^{*}>\max (1,1-\tilde{r} / D)$. This readily implies the uniqueness of the root $\rho^{*}$.

Now, we analyze the existence of $\rho^{*}$. If $\tilde{r}>0$, then the existence of $\rho^{*}>1$ follows from the fact that $F(1, D)=(\tilde{r}-r) / D<0$ by $(2)$, and $\lim _{\rho \rightarrow+\infty} F(\rho, D)=+\infty$. Furthermore, $\rho^{*}>1$ exists for all $D>0$. Now, consider the case $\tilde{r}<0<r$ (equivalently, $f>r$ ). A direct evaluation shows that

$$
F(1-\tilde{r} / D, D)=\frac{1}{D}\left(-\tilde{r}-r+\frac{r \tilde{r}}{D}\right)=\frac{1}{D}\left(f-2 r+\frac{r(r-f)}{D}\right) .
$$


If $r<f<2 r$, we have that $F(1-\tilde{r} / D, D)<0$ for all $D>0$, hence $\rho^{*}$ exists for all $D>0$. If $f>2 r$, then $F(1-\tilde{r} / D, D)<0$ if and only if

$$
D<D_{\max }:=\frac{r(f-r)}{f-2 r}=\frac{r \tilde{r}}{r+\tilde{r}} .
$$

[Note that $D_{\max }$ is half of the harmonic mean of $r$ and $\tilde{r}$.] Hence, if $f>2 r$, a positive steady state exists only for $D<D_{\max }$.

Differentiating $F$ with respect to $D$, we obtain

$$
F_{D}\left(\rho^{*}, D\right)=-\frac{\tilde{r}\left(\rho^{*}\right)^{2}-r \rho^{*}}{D^{2}}=\frac{\left(\rho^{*}\right)^{3}-\left(\rho^{*}\right)^{2}+\rho^{*}-1}{D} .
$$

Since $\rho^{*}>1$, we conclude that $F_{D}\left(\rho^{*}, D\right)>0$. Using the Implicit Function Theorem, we conclude that there is a unique, smooth and decreasing function $\rho^{*}(D)$ such that $F\left(\rho^{*}(D), D\right)=0$. A direct calculation shows that $\rho^{*}(D) \rightarrow 1$ as $D \rightarrow+\infty$ in the case $f<2 r$, and $\rho^{*}(D) \rightarrow \frac{f-r}{r}>1$ as $D \rightarrow D_{\max }$ in the case $f>2 r$.

Finally, since system (1) is a strongly monotone system, it can be shown that all positive solutions converge to the unique steady state $\left(u_{1}^{*}, u_{2}^{*}\right)$, see Proposition 4.4 .1 in [44].

\section{B Appendix: Predator-prey model}

Recall the celebrated Lotka-Volterra predator-prey model:

$$
\begin{aligned}
& \frac{\mathrm{d} x}{\mathrm{~d} t}=x(r-c y), \\
& \frac{\mathrm{d} y}{\mathrm{~d} t}=y(c x-d),
\end{aligned}
$$

where $x$ and $y$ are the densities of prey and predator respectively, and $r, c$ and $d$ are positive constants, representing the per capita growth rate of the prey, the capture rate, and the predator's mortality rate, respectively. Rescaling time by $t^{\prime}=c t$, $r^{\prime}=\frac{r}{c}$, and $d^{\prime}=\frac{d}{c}$ (and suppressing the prime notation) simplifies the system to

$$
\begin{aligned}
& \frac{\mathrm{d} x}{\mathrm{~d} t}=x(r-y), \\
& \frac{\mathrm{d} y}{\mathrm{~d} t}=y(x-d) .
\end{aligned}
$$

It is straightforward to show that there is a unique positive steady state $E^{*}=$ $\left(x^{*}, y^{*}\right)=(d, r)$, which is neutrally stable. In fact, the system is conservative and has first integral

$$
V(x, y)=\int_{x^{*}}^{x}\left(1-\frac{x^{*}}{s}\right) \mathrm{d} s+\int_{y^{*}}^{y}\left(1-\frac{y^{*}}{s}\right) \mathrm{d} s,
$$

which is easily verified by a straightforward calculation. 


\section{B.1 The effect of fishing}

When the prey and predator are harvested at rates $f_{x}$ and $f_{y}$ respectively, we get the system

$$
\begin{aligned}
& \frac{\mathrm{d} x}{\mathrm{~d} t}=x(r-y)-f_{x} x \\
& \frac{\mathrm{d} y}{\mathrm{~d} t}=y(x-d)-f_{y} y .
\end{aligned}
$$

Note that (21) takes the form of the classical Lotka-Volterra system

$$
\begin{aligned}
& \frac{\mathrm{d} x}{\mathrm{~d} t}=x(\tilde{r}-y), \\
& \frac{\mathrm{d} y}{\mathrm{~d} t}=y(x-\tilde{d}),
\end{aligned}
$$

where

$$
\tilde{r}:=r-f_{x}<r \quad \text { and } \quad \tilde{d}:=d+f_{y}>d .
$$

As in the case of the single-species model, we assume that the prey harvesting rate is small enough to avoid population collapse, $f_{x}<r$. Then $\tilde{r}>0$ and hence system (21) has a unique, neutrally stable positive steady state $\tilde{E}=(\tilde{d}, \tilde{r})$. This system is also conservative.

\section{Appendix: Predator-prey MPA-FG model}

\section{C.1 Predator moves, but prey is sessile}

Here we investigate the case where $D_{x}=0$ and $D_{y}>0$. Then

$$
E_{y}^{*}=\left(x_{1}^{*}, y_{1}^{*}, x_{2}^{*}, y_{2}^{*}\right)=\left(d+D_{y}\left(1-\left(\rho_{y}^{0}\right)^{-1}\right), r, \tilde{d}+D_{y}\left(1-\rho_{y}^{0}\right), \tilde{r}\right)
$$

where $\rho_{y}^{0}>1$ was defined in $(10)$, is the unique positive steady state if and only if

$$
0 \leq D_{y}<\frac{\tilde{r} \tilde{d}}{r-\tilde{r}} .
$$

Theorem 2. Suppose that $D_{x}=0$ and $D_{y} \in \mathcal{I}_{y}:=\left(0, \frac{\tilde{r} \tilde{d}}{r-\tilde{r}}\right)$. Then

1. $E_{y}^{*}$ attracts all positive solutions of $(7)$.

(a) If $\tilde{r} \tilde{d} \leq r d, E_{y}^{*}$ is linearly stable for all $D_{y} \in \mathcal{I}_{y}$.

(b) If $\tilde{r} \tilde{d}>r d, E_{y}^{*}$ is linearly stable for all $D_{y} \in \mathcal{I}_{y} \backslash\left\{D_{y}^{*}:=\frac{\tilde{r} \tilde{d}-r d}{2(r-\tilde{r})}\right\}$, and neutrally stable when $D_{y}=D_{y}^{*}$ due to the linearization at $E_{y}^{*}$ having a pair of purely imaginary eigenvalues, in addition to two eigenvalues with negative real part. 
2. The steady-state prey ratio $x_{1}^{*} / x_{2}^{*}$ is increasing with $D_{y}$. It is less than one if $D_{y}<(\tilde{d}-d) /\left(\rho_{y}-\rho_{y}^{-1}\right)=: D_{y}^{\text {crit }}$, but larger than or equal to 1 otherwise, and it tends to infinity as $D_{y} \rightarrow \frac{\tilde{r} \tilde{d}}{r-\tilde{r}}$. Consequently, increasing the diffusivity $D_{y}$ is equalizing for the prey if $D_{y}$ remains below $D_{y}^{\text {crit }}$. But it is not equalizing when $D_{y}$ exceeds this bound.

3. The steady-state predator ratio $y_{1}^{*} / y_{2}^{*}=r / \tilde{r}$ is constant in $D_{y}$.

Proof. The proofs of the second and third statements follow immediately from an inspection of the steady state expressions as functions of $D_{y}$ in (11). Note in particular that $(\tilde{d}-d) /\left(\rho_{y}^{0}-\left(\rho_{y}^{0}\right)^{-1}\right) \in \mathcal{I}_{y}$. To prove the global attractivity in the first statement we will start by constructing a weak Lyapunov function, and then conclude the proof by an application of LaSalle's invariance principle.

Define

$$
\begin{aligned}
V\left(x_{1}, y_{1}, x_{2}, y_{2}\right) & =\int_{x_{1}^{*}}^{x_{1}}\left(1-\frac{x_{1}^{*}}{s}\right) d s+\int_{y_{1}^{*}}^{y_{1}}\left(1-\frac{y_{1}^{*}}{s}\right) d s \\
& +C\left(\int_{x_{2}^{*}}^{x_{2}}\left(1-\frac{x_{2}^{*}}{s}\right) d s+\int_{y_{2}^{*}}^{y_{2}}\left(1-\frac{y_{2}^{*}}{s}\right) d s\right),
\end{aligned}
$$

where $C>0$ is a constant determined below. Note that $V$ is positive semi-definite $\operatorname{in} \operatorname{int}\left(\mathbb{R}_{+}^{4}\right)$, and zero if and only if $\left(x_{1}, y_{1}, x_{2}, y_{2}\right)=\left(x_{1}^{*}, y_{1}^{*}, x_{2}^{*}, y_{2}^{*}\right)$. It is also a proper function in $\operatorname{int}\left(\mathbb{R}_{+}^{4}\right)$.

We calculate the derivative along a solution of $(7)$ in $\operatorname{int}\left(\mathbb{R}_{+}^{4}\right)$ :

$$
\begin{aligned}
& \dot{V}=\left(r-y_{1}\right)\left(x_{1}-x_{1}^{*}\right)+\left(x_{1}-d\right)\left(y_{1}-y_{1}^{*}\right)+D_{y}\left(\frac{y_{2}}{y_{1}}-1\right)\left(y_{1}-y_{1}^{*}\right) \\
& +C\left(\left(\tilde{r}-y_{2}\right)\left(x_{2}-x_{2}^{*}\right)+\left(x_{2}-\tilde{d}\right)\left(y_{2}-y_{2}^{*}\right)+D_{y}\left(\frac{y_{1}}{y_{2}}-1\right)\left(y_{2}-y_{2}^{*}\right)\right) \\
& =-\left(y_{1}-y_{1}^{*}\right)\left(x_{1}-x_{1}^{*}\right)+\left(x_{1}-x_{1}^{*}\right)\left(y_{1}-y_{1}^{*}\right) \\
& +D_{y}\left(1-\left(\rho_{y}^{0}\right)^{-1}\right)\left(y_{1}-y_{1}^{*}\right)+D_{y}\left(\frac{y_{2}}{y_{1}}-1\right)\left(y_{1}-y_{1}^{*}\right) \\
& +C\left(-\left(y_{2}-y_{2}^{*}\right)\left(x_{2}-x_{2}^{*}\right)+\left(x_{2}-x_{2}^{*}\right)\left(y_{2}-y_{2}^{*}\right)\right) \\
& +C\left(D_{y}\left(1-\rho_{y}^{0}\right)\left(y_{2}-y_{2}^{*}\right)+D_{y}\left(\frac{y_{1}}{y_{2}}-1\right)\left(y_{2}-y_{2}^{*}\right)\right) \\
& =D_{y}\left(y_{1}-y_{1}^{*}\right)\left(\frac{y_{2}}{y_{1}}-\left(\rho_{y}^{0}\right)^{-1}\right)+C D_{y}\left(y_{2}-y_{2}^{*}\right)\left(\frac{y_{1}}{y_{2}}-\rho_{y}^{0}\right) \\
& =D_{y} \frac{\frac{y_{1}}{y_{2}}-\rho_{y}^{0}}{\rho_{y}^{0} \frac{y_{1}}{y_{2}}}\left(-\left(y_{1}-y_{1}^{*}\right)+C \rho_{y}^{0} \frac{y_{1}}{y_{2}}\left(y_{2}-y_{2}^{*}\right)\right)
\end{aligned}
$$

Set

$$
C=\left(\rho_{y}^{0}\right)^{-1},
$$

and after first simplifying and factoring out $y_{2}^{*}$ in the last factor in parentheses, we find that:

$$
\dot{V}=D_{y} \frac{\frac{y_{1}}{y_{2}}-\rho_{y}^{0}}{\rho_{y}^{0} \frac{y_{1}}{y_{2}}} y_{2}^{*}\left(\frac{y_{1}^{*}}{y_{2}^{*}}-\frac{y_{1}}{y_{2}}\right)=-D_{y} \frac{\left(\frac{y_{1}}{y_{2}}-\rho_{y}^{0}\right)^{2}}{\rho_{y}^{0} \frac{y_{1}}{y_{2}}} y_{2}^{*} \leq 0
$$


which implies that all positive solutions remain bounded, and bounded away from the boundary of $\operatorname{int}\left(\mathbb{R}_{+}^{4}\right.$ ) (both by properness of $V$ ). Note also that $\dot{V}=0$ if and only if

$$
\frac{y_{1}}{y_{2}}=\rho_{y}^{0}
$$

and by LaSalle's invariance principle, every positive solution converges to the largest invariant set contained in the set where (26) holds. Let us consider the dynamics of the ratio $y_{1}(t) / y_{2}(t)$ along positive solutions of system $(7)$ :

$$
\frac{d}{d t}\left(\frac{y_{1}(t)}{y_{2}(t)}\right)=\frac{y_{1}(t)}{y_{2}(t)}\left(x_{1}(t)-x_{2}(t)-(d-\tilde{d})\right)+D_{y}\left(1-\left(\frac{y_{1}(t)}{y_{2}(t)}\right)^{2}\right)
$$

Thus, if a solution is such that it is contained in the set where (26) holds, then the left-hand side of the last equation must be zero, and we can set $y_{1}(t) / y_{2}(t)=\rho_{y}^{0}$ in the right-hand side. Consequently, the difference of $x_{1}(t)$ and $x_{2}(t)$ along such a positive solution is constant:

$$
x_{1}(t)-x_{2}(t)=(d-\tilde{d})+D_{y}\left(\rho_{y}^{0}-\left(\rho_{y}^{0}\right)^{-1}\right)
$$

Moreover, when (26) holds, it follows that:

$$
\frac{\dot{x}_{1}}{x_{1}}-\rho_{y}^{0} \frac{\dot{x}_{2}}{x_{2}}=0
$$

and hence $x_{1} x_{2}^{-\rho_{y}^{0}}$ is constant as well. Together with (27), this implies that $x_{1}(t)$ and $x_{2}(t)$ must remain constant, both necessarily positive. Then the system equations (7a) and (7c) imply that $y_{1}(t)$ and $y_{2}(t)$ must remain constant, also both positive. Thus, any solution satisfying (26) must coincide with the unique positive steady state $E^{*}$. In summary, we have shown that every positive solution must converge to the positive steady state.

Finally, we apply the Routh-Hurwitz Theorem to assess the local stability properties of $E_{y}^{*}$. The linearization of $(7)$ at $E_{y}^{*}$ is:

$$
\left(\begin{array}{cccc}
0 & -x_{1}^{*} & 0 & 0 \\
y_{1}^{*} & -\left(\rho_{y}^{0}\right)^{-1} D_{y} & 0 & D_{y} \\
0 & 0 & 0 & -x_{2}^{*} \\
0 & D_{y} & y_{2}^{*} & -\rho_{y}^{0} D_{y}
\end{array}\right)
$$

which has characteristic polynomial:

$$
\lambda^{4}+a_{3} \lambda^{3}+a_{2} \lambda^{2}+a_{1} \lambda+a_{0}
$$

where

$$
\begin{aligned}
& a_{3}=D_{y}\left(\rho_{y}^{0}+\left(\rho_{y}^{0}\right)^{-1}\right) \\
& a_{2}=x_{1}^{*} y_{1}^{*}+x_{2}^{*} y_{2}^{*} \\
& a_{1}=D_{y}\left(\left(\rho_{y}^{0}\right)^{-1} x_{2}^{*} y_{2}^{*}+\rho_{y}^{0} x_{1}^{*} y_{1}^{*}\right) \\
& a_{0}=x_{1}^{*} x_{2}^{*} y_{1}^{*} y_{2}^{*}
\end{aligned}
$$


The Routh-Hurwitz Theorem states that all the roots of this polynomial have negative real real part if and only if the four entries of the first column of the RouthHurwitz table are positive:

$$
a_{0}>0, a_{3}>0, a_{2} a_{3}-a_{1}>0, \text { and } a_{1} a_{2} a_{3}-\left(a_{1}^{2}+a_{0} a_{3}^{2}\right)>0 .
$$

The above expressions for $a_{0}$ and $a_{3}$ clearly show that the first two inequalities hold because $E_{y}^{*}$ is a positive steady state if $D_{y} \in \mathcal{I}_{y}$. To check the third inequality, we use the definition of $E_{y}^{*}$ in (11), and invoke the definition of $\rho_{y}^{0}=r / \tilde{r}$, to express $a_{2} a_{3}-a_{1}$ in terms of the 4 model parameters $r, d, \tilde{r}$ and $\tilde{d}$. Some manipulations reveal that

$$
a_{2} a_{3}-a_{1}=\frac{D_{y}}{r \tilde{r}}\left(r \tilde{r}(\tilde{r} d+r \tilde{d})-D_{y}(r-\tilde{r})^{2}(r+\tilde{r})\right)
$$

Note that the term in parentheses is linearly decreasing in $D_{y}$. We evaluate it when $D_{y}$ equals the upper bound of $\mathcal{I}_{y}$, i.e. for $D_{y}=\tilde{r} \tilde{d} /(r-\tilde{r})$, and find that it equals $\tilde{r}^{2}(r d+\tilde{r} \tilde{d})$, which is positive. Hence,

$$
a_{2} a_{3}-a_{1}>0 \text { for all } D_{y} \in \mathcal{I}_{y} .
$$

To check the sign of the fourth quantity from the Routh-Hurwitz Theorem, we also express it in terms of the 4 model parameters. After some tedious calculations, we find that it is a product of two quadratic expressions in $D_{y}$ :

$$
a_{1} a_{2} a_{3}-\left(a_{1}^{2}+a_{0} a_{3}^{2}\right)=D_{y}^{2}\left(2(r-\tilde{r}) D_{y}+(r d-\tilde{r} \tilde{d})\right)^{2} \geq 0 \text { if } D_{y} \in \mathcal{I}_{y} .
$$

Notice that the above inequality is strict for all $D_{y} \in \mathcal{I}_{y}$ if $r d \geq \tilde{r} \tilde{d}$. However, if $r d<\tilde{r} \tilde{d}$, it is zero if and only if $D_{y}=D_{y}^{*}$, and in this case, the Routh-Hurwitz criterion fails because the fourth row in the Routh-Hurwitz table consists of zeros only. To further explore this case, we assume that $r d<\tilde{r} \tilde{d}$ and $D_{y}=D_{y}^{*}$, and construct an auxiliary polynomial:

$$
\frac{a_{2} a_{3}-a_{1}}{a_{3}} \lambda^{2}+a_{0}
$$

and note that this polynomial has 2 purely imaginary roots (the coefficient of $\lambda^{2}$ is positive, and so is $a_{0}$ ). Consequently, the characteristic polynomial has two purely imaginary roots. Furthermore, the derivative of the auxiliary polynomial with respect to $\lambda$ is $2\left(a_{2} a_{3}-a_{1}\right) / a_{3} \lambda$, which is used to replace the zero in the 4th entry of the first column of the Routh-Hurwitz table. Completing the table in the usual way yields a table with only positive entries in the first column, and thus the characteristic polynomial has no roots in the open right-half plane. Since it has two roots on the imaginary axis, it must also have two roots with negative real part.

\section{C.2 Prey moves, but predator is sessile}

Here we investigate the case where $D_{x}>0$ and $D_{y}=0$. Then

$$
E_{x}^{*}=\left(x_{1}^{*}, y_{1}^{*}, x_{2}^{*}, y_{2}^{*}\right)=\left(d, r+D_{x}\left(\left(\rho_{x}^{0}\right)^{-1}-1\right), \tilde{d}, \tilde{r}+D_{x}\left(\rho_{x}^{0}-1\right)\right)
$$


where $\rho_{x}^{0}<1$ was defined in (9), is the unique positive steady state if and only if

$$
0 \leq D_{x}<\frac{\tilde{r} \tilde{d}}{\tilde{d}-d}
$$

Theorem 3. Suppose that $D_{x} \in \mathcal{I}_{x}:=\left(0, \frac{\tilde{r} \tilde{d}}{\tilde{d}-d}\right)$ and $D_{y}=0$. Then

1. $E_{x}^{*}$ attracts all positive solutions of $(7)$.

(a) If $\tilde{r} \tilde{d} \leq r d, E_{x}^{*}$ is linearly stable for all $D_{x} \in \mathcal{I}_{x}$.

(b) If $\tilde{r} \tilde{d}>r d, E_{x}^{*}$ is linearly stable for all $D_{x} \in \mathcal{I}_{x} \backslash\left\{D_{x}^{*}:=\frac{\tilde{r} \tilde{d}-r d}{2(\tilde{d}-d)}\right\}$, and neutrally stable when $D_{x}=D_{x}^{*}$ due to the linearization at $E_{x}^{*}$ having a pair of purely imaginary eigenvalues, in addition to two eigenvalues with negative real part.

2. The steady state prey ratio $x_{1}^{*} / x_{2}^{*}=d / \tilde{d}$ is constant in $D_{x}$.

3. The steady state predator ratio $y_{1}^{*} / y_{2}^{*}$ increasing with $D_{x}$. It is larger than one for all $D_{x} \in \mathcal{I}_{x}$, and it increases to infinity as $D_{x}$ approaches $\tilde{r} \tilde{d} /(\tilde{d}-d)$. Consequently, increasing the diffusivity $D_{x}$ is never equalizing for the predator.

Proof. This proof is very similar to that of Theorem 2, hence it will only be presented in abbreviated form. The proofs of the second and third statements follow immediately from an inspection of the steady state expressions as functions of $D_{x}$ in $E_{x}^{*}$. To prove the global attractivity, we define

$$
\begin{aligned}
V\left(x_{1}, y_{1}, x_{2}, y_{2}\right) & =\int_{x_{1}^{*}}^{x_{1}}\left(1-\frac{x_{1}^{*}}{s}\right) d s+\int_{y_{1}^{*}}^{y_{1}}\left(1-\frac{y_{1}^{*}}{s}\right) d s \\
& +C^{\prime}\left(\int_{x_{2}^{*}}^{x_{2}}\left(1-\frac{x_{2}^{*}}{s}\right) d s+\int_{y_{2}^{*}}^{y_{2}}\left(1-\frac{y_{2}^{*}}{s}\right) d s\right),
\end{aligned}
$$

where $C^{\prime}>0$ is a constant to be determined below. Note that $V$ is positive semidefinite in $\operatorname{int}\left(\mathbb{R}_{+}^{4}\right)$, and zero if and only if $\left(x_{1}, y_{1}, x_{2}, y_{2}\right)=\left(x_{1}^{*}, y_{1}^{*}, x_{2}^{*}, y_{2}^{*}\right)$. It is also a proper function in int $\left(\mathbb{R}_{+}^{4}\right)$.

Similar calculations as in the previous section show that the derivative of $V$ satisfies:

$$
\begin{aligned}
\dot{V} & =\frac{D_{x}\left(\frac{x_{2}}{x_{1}}-\left(\rho_{x}^{0}\right)^{-1}\right)}{\frac{x_{2}}{x_{1}}}\left(\left(x_{1}-x_{1}^{*}\right) \frac{x_{2}}{x_{1}}-C^{\prime} \rho_{x}^{0}\left(x_{2}-x_{2}^{*}\right)\right) \\
& =\frac{-x_{1}^{*} D_{x}\left(\frac{x_{2}}{x_{1}}-\left(\rho_{x}^{0}\right)^{-1}\right)^{2}}{\frac{x_{2}}{x_{1}}} \leq 0,
\end{aligned}
$$

by choosing $C^{\prime}=\left(\rho_{x}^{0}\right)^{-1}$. We find that $\dot{V}=0$ if and only if $\frac{x_{2}}{x_{1}}=\left(\rho_{x}^{0}\right)^{-1}$, and by LaSalle's invariance principle, every positive solution converges to the largest invariant set contained in the set where this identity holds. Consider a solution with 
$\frac{x_{2}}{x_{1}} \equiv\left(\rho_{x}^{0}\right)^{-1}$. Differentiating the ratio $\frac{x_{2}}{x_{1}}$ and setting the derivative equal to zero, we find that

$$
0 \equiv\left(\rho_{x}^{0}\right)^{-1}\left(\tilde{r}-r+y_{1}-y_{2}\right)+D_{x}\left(1-\left(\rho_{x}^{0}\right)^{-2}\right),
$$

hence $y_{1}-y_{2}$ remains constant along such solution. In addition, we have that

$$
\frac{\dot{y}_{1}}{y_{1}}-\rho_{x}^{0} \frac{\dot{y}_{2}}{y_{2}} \equiv 0
$$

therefore the product $y_{1} y_{2}^{-\rho_{x}^{0}}$ remains constant as well. Combining these two results, we conclude that both $y_{1}$ and $y_{2}$ remain constant, hence $x_{1} \equiv x_{1}^{*}$ and $x_{2} \equiv x_{2}^{*}$. From this, it follows that $y_{1} \equiv y_{1}^{*}$ and $y_{2} \equiv y_{2}^{*}$. Thus, the only solution with $\frac{x_{1}}{x_{2}} \equiv \rho_{x}^{0}$ is the positive steady state which attracts all positive solutions.

Finally, we apply the Routh-Hurwitz Theorem to assess the local stability properties of $E_{x}^{*}$. The linearization of $(7)$ at $E_{x}^{*}$ is:

$$
\left(\begin{array}{cccc}
-\left(\rho_{x}^{0}\right)^{-1} D_{x} & -x_{1}^{*} & D_{x} & 0 \\
y_{1}^{*} & 0 & 0 & 0 \\
D_{x} & 0 & -\rho_{x}^{0} D_{x} & -x_{2}^{*} \\
0 & 0 & y_{2}^{*} & 0
\end{array}\right)
$$

which is similar by permuting first and second, and third and fourth variables to:

$$
\left(\begin{array}{cccc}
0 & y_{1}^{*} & 0 & 0 \\
-x_{1}^{*} & -\left(\rho_{x}^{0}\right)^{-1} D_{x} & 0 & D_{x} \\
0 & 0 & 0 & y_{2}^{*} \\
0 & D_{x} & -x_{2}^{*} & -\rho_{x}^{0} D_{x}
\end{array}\right)
$$

which by the proof in the previous section has characteristic polynomial:

$$
\lambda^{4}+a_{3} \lambda^{3}+a_{2} \lambda^{2}+a_{1} \lambda+a_{0}
$$

where

$$
\begin{aligned}
& a_{3}=D_{x}\left(\rho_{x}^{0}+\left(\rho_{x}^{0}\right)^{-1}\right) \\
& a_{2}=x_{1}^{*} y_{1}^{*}+x_{2}^{*} y_{2}^{*} \\
& a_{1}=D_{x}\left(\left(\rho_{x}^{0}\right)^{-1} x_{2}^{*} y_{2}^{*}+\rho_{x}^{0} x_{1}^{*} y_{1}^{*}\right) \\
& a_{0}=x_{1}^{*} x_{2}^{*} y_{1}^{*} y_{2}^{*}
\end{aligned}
$$

The Routh-Hurwitz Theorem states that all the roots of this polynomial have negative real real part if and only if the four entries of the first column of the RouthHurwitz table are positive:

$$
a_{0}>0, a_{3}>0, a_{2} a_{3}-a_{1}>0 \text {, and } a_{1} a_{2} a_{3}-\left(a_{1}^{2}+a_{0} a_{3}^{2}\right)>0 .
$$

As before $a_{0}$ and $a_{3}$ are positive for all $D_{x} \in \mathcal{I}_{x}$. Furthermore, some algebra shows that:

$$
a_{2} a_{3}-a_{1}=\frac{D_{x}}{d \tilde{d}}\left(d \tilde{d}(\tilde{r} d+r \tilde{d})+D_{x}(\tilde{d}-d)^{2}(\tilde{d}+d)\right)>0
$$


for all non-negative $D_{x}$. Finally,

$$
a_{1} a_{2} a_{3}-\left(a_{1}^{2}+a_{0} a_{3}^{2}\right)=D_{x}^{2}\left(2(\tilde{d}-d) D_{x}-(\tilde{r} \tilde{d}-r d)\right)^{2} \geq 0 \text { if } D_{x} \in \mathcal{I}_{x}
$$

This inequality is strict for all $D_{x} \in \mathcal{I}_{x}$ if $r d \geq \tilde{r} \tilde{d}$. However, if $r d<\tilde{r} \tilde{d}$, it is zero if and only if $D_{x}=D_{x}^{*}$, and in this case, the Routh-Hurwitz criterion fails because the fourth row in the Routh-Hurwitz table consists of zeros only. Continuing as in the proof in the previous section we see that if $r d<\tilde{r} \tilde{d}$ and $D_{x}=D_{x}^{*}$, then the characteristic polynomial has a pair of purely imaginary roots, in addition to a pair of roots with negative real part.

\section{C.3 Both predator and prey move}

Here we consider the case where both $D_{x}>0$ and $D_{y}>0$. We start by establishing that there always is a unique positive steady state.

Theorem 4. Suppose that $D_{x}>0$ and $D_{y}>0$. Then (7) has a unique, positive steady-state $E_{x y}^{*}=\left(x_{1}^{*}, y_{1}^{*}, x_{2}^{*}, y_{2}^{*}\right)$. Moreover, the prey and predator steady state ratios $\rho_{x}:=x_{1}^{*} / x_{2}^{*}$ and $\rho_{y}:=y_{1}^{*} / y_{2}^{*}$ satisfy:

$$
\rho_{y}>1, \text { and } \rho_{x} \begin{cases}<1, & \text { if } D_{y}<D_{y}^{c r i t}:=\frac{d-\tilde{d}}{\rho_{y}^{0}-\left(\rho_{y}^{0}\right)^{-1}} \\ =1, & \text { if } D_{y}=D_{y}^{c r i t} \\ >1, & \text { if } D_{y}>D_{y}^{c r i t}\end{cases}
$$

If $\rho_{x} \leq 1$, or equivalently if $D_{y} \leq D_{y}^{\text {crit }}$, then $E_{x y}^{*}$ is locally asymptotically stable.

Proof. We seek a positive solution $\left(x_{1}, y_{1}, x_{2}, y_{2}\right)$ to the system

$$
\begin{aligned}
& 0=x_{1}\left(r-y_{1}\right)+D_{x}\left(x_{2}-x_{1}\right) \\
& 0=y_{1}\left(x_{1}-d\right)+D_{y}\left(y_{2}-y_{1}\right) \\
& 0=x_{2}\left(\tilde{r}-y_{2}\right)+D_{x}\left(x_{1}-x_{2}\right) \\
& 0=y_{2}\left(x_{2}-\tilde{d}\right)+D_{y}\left(y_{1}-y_{2}\right) .
\end{aligned}
$$

Defining the ratios $\rho_{x}:=x_{1} / x_{2}$ and $\rho_{y}:=y_{1} / y_{2}$, we can uniquely express all state variables in terms of $\rho_{x}$ and $\rho_{y}$ as follows:

$$
\begin{aligned}
& y_{1}=r+D_{x}\left(1 / \rho_{x}-1\right), \\
& x_{1}=d-D_{y}\left(1 / \rho_{y}-1\right), \\
& y_{2}=\tilde{r}+D_{x}\left(\rho_{x}-1\right), \\
& x_{2}=\tilde{d}-D_{y}\left(\rho_{y}-1\right) .
\end{aligned}
$$

These values, in turn, must satisfy

$$
\begin{aligned}
\rho_{x} & =\frac{d-D_{y}\left(1 / \rho_{y}-1\right)}{\tilde{d}-D_{y}\left(\rho_{y}-1\right)}=\frac{\left(d+D_{y}\right) \rho_{y}-D_{y}}{\rho_{y}\left(\tilde{d}+D_{y}-D_{y} \rho_{y}\right)}:=F\left(\rho_{y}\right), \\
\rho_{y} & =\frac{r+D_{x}\left(1 / \rho_{x}-1\right)}{\tilde{r}+D_{x}\left(\rho_{x}-1\right)}=\frac{\left(r-D_{x}\right) \rho_{x}+D_{x}}{\rho_{x}\left(\tilde{r}-D_{x}+D_{x} \rho_{x}\right)}:=G\left(\rho_{x}\right) .
\end{aligned}
$$


Positive equilibria correspond to solutions of the system $\rho_{x}=F\left(\rho_{y}\right), \rho_{y}=G\left(\rho_{x}\right)$ in the region where the following inequalities are satisfied

$\left(d+D_{y}\right) \rho_{y}-D_{y}>0, \quad \tilde{d}+D_{y}-D_{y} \rho_{y}>0, \quad\left(r-D_{x}\right) \rho_{x}+D_{x}>0, \quad \tilde{r}-D_{x}+D_{x} \rho_{x}>0$.

We will study this system graphically. First, consider the curve $\rho_{x}=F\left(\rho_{y}\right)$ in the first quadrant. It is a graph of an increasing function starting at the point $\left(\rho_{x}, \rho_{y}\right)=\left(0, \frac{D_{y}}{d+D_{y}}\right)$ and approaching a horizontal asymptote $\rho_{y}=\frac{\tilde{d}+D_{y}}{D_{y}}$. Note the relation $\frac{D_{y}}{d+D_{y}}<1<\frac{\tilde{d}+D_{y}}{D_{y}}$. Also note that the quantities $\left(d+D_{y}\right) \rho_{y}-D_{y}$ and $\tilde{d}+D_{y}-D_{y} \rho_{y}$ are simultaneously positive if and only if $\rho_{y} \in\left(\frac{D_{y}}{d+D_{y}}, \frac{\tilde{d}+D_{y}}{D_{y}}\right)$.

Second, the shape of the curve $\rho_{y}=G\left(\rho_{x}\right)$ varies slightly with the value of $D_{x}$. We recall that $0<\tilde{r}<r$.

1. If $D_{x} \leq \tilde{r}<r$, the curve $\rho_{y}=G\left(\rho_{x}\right)$ in the first quadrant is a graph of a decreasing function with a vertical asymptote at $\rho_{x}=0$ and a horizontal asymptote $\rho_{y}=0$. Therefore, the system $\rho_{x}=F\left(\rho_{y}\right), \rho_{y}=G\left(\rho_{x}\right)$ has a unique positive solution. Furthermore, it is clear that this solution satisfies (34).

2. If $\tilde{r}<D_{x} \leq r$, the curve $\rho_{y}=G\left(\rho_{x}\right)$ in the first quadrant is a graph of a decreasing function with a vertical asymptote at $\rho_{x}=\frac{D_{x}-\tilde{r}}{D_{x}}$ and a horizontal asymptote $\rho_{y}=0$. Again, the system $\rho_{x}=F\left(\rho_{y}\right), \rho_{y}=G\left(\rho_{x}\right)$ has a unique positive solution which satisfies (34).

3. If $\tilde{r}<r<D_{x}$, the curve $\rho_{y}=G\left(\rho_{x}\right)$ in the first quadrant is graph of a decreasing function with a vertical asymptote at $\rho_{x}=\frac{D_{x}-\tilde{r}}{D_{x}}<1$ and a zero at $\rho_{x}=\frac{D_{x}}{D_{x}-r}>1$. Again, the system $\rho_{x}=F\left(\rho_{y}\right), \rho_{y}=G\left(\rho_{x}\right)$ has a unique positive solution which satisfies (34).

We conclude that for any positive combination $\left(D_{x}, D_{y}\right)$, the system admits a unique positive equilibrium $E_{x y}^{*}=\left(x_{1}, y_{1}, x_{2}, y_{2}\right)$.

In all three cases, we have that $F(1)=d / \tilde{d}<1$ with $F$ increasing and $G(1)=$ $r / \tilde{r}>1$ with $G$ decreasing, therefore the point of intersection $\left(\rho_{x}, \rho_{y}\right)$ always occurs in the region where $\rho_{y}>1$. Whether or not $\rho_{x}$ is less or larger than 1 , is more intricate:

- If $r / \tilde{r}=G(1) \geq\left(\tilde{d}+D_{y}\right) / D_{y}$, then $r / \tilde{r}$ is outside the domain of the function $F$ in the first quadrant, and hence then $\rho_{x}>1$ in all three cases. But the condition $r / \tilde{r}>\left(\tilde{d}+D_{y}\right) / D_{y}$ is equivalent to:

$$
D_{y} \geq \frac{\tilde{r} \tilde{d}}{r-\tilde{r}}
$$

- On the other hand, if $r / \tilde{r}=G(1)<\left(\tilde{d}+D_{y}\right) / D_{y}$, or equivalently if $D_{y}<$ $\tilde{r} \tilde{d} /(r-\tilde{r})$, then $r / \tilde{r}$ does belong to the domain of the function $F$ in the first quadrant in all three cases. Since $F$ is invertible (as it is increasing there), it follows that $\rho_{x}$ satisfies the following:

$$
\rho_{x}\left\{\begin{array}{l}
>1 \text { if and only if } F(r / \tilde{r})>1 \\
=1 \text { if and only if } F(r / \tilde{r})=1 \\
<1 \text { if and only if } F(r / \tilde{r})<1
\end{array}\right.
$$


Using the definition of the function $F$, it is easily verified that this is equivalent to

$$
\rho_{x}\left\{\begin{array}{l}
>1 \text { if and only if } D_{y}>D_{y}^{c r i t} \\
=1 \text { if and only if } D_{y}=D_{y}^{c r i t} \\
<1 \text { if and only if } D_{y}<D_{y}^{c r i t}
\end{array}\right.
$$

where $D_{y}^{\text {crit }}=(\tilde{d}-d) /\left(\rho_{y}^{0}-\left(\rho_{y}^{0}\right)^{-1}\right)$.

Since $D_{y}^{\text {crit }}<\tilde{r} \tilde{d} /(r-\tilde{r})$, we can summarize the location of $\rho_{x}$ with respect to 1 , in terms of the value of $D_{y}$ : For all positive $D_{x}$ and $D_{y}$, we have that the prey steady state ratio satisfies

$$
\rho_{x}\left\{\begin{array}{l}
>1 \text { if and only if } D_{y}>D_{y}^{\text {crit }} \\
=1 \text { if and only if } D_{y}=D_{y}^{\text {crit }} \\
<1 \text { if and only if } D_{y}<D_{y}^{\text {crit }}
\end{array}\right.
$$

The Jacobian matrix at $E_{x y}^{*}$ takes the form

$$
\left(\begin{array}{cccc}
r-y_{1}-D_{x} & -x_{1} & D_{x} & 0 \\
y_{1} & x_{1}-d-D_{y} & 0 & D_{y} \\
D_{x} & -0 & \tilde{r}-y_{2}-D_{x} & -x_{2} \\
0 & D_{y} & y_{2} & x_{2}-\tilde{d}-D_{y}
\end{array}\right)
$$

which after substituting the equilibrium relationships and using the previously defined ratios $\rho_{x}=x_{1} / x_{2}$ and $\rho_{y}=y_{1} / y_{2}$ simplifies to

$$
\left(\begin{array}{cccc}
-D_{x} \rho_{x}^{-1} & -x_{1} & D_{x} & 0 \\
y_{1} & -D_{y} \rho_{y}^{-1} & 0 & D_{y} \\
D_{x} & -0 & -D_{x} \rho_{x} & -x_{2} \\
0 & D_{y} & y_{2} & -D_{y} \rho_{y}
\end{array}\right)
$$

The characteristic polynomial of this matrix is given by

$$
p(\lambda)=\lambda^{4}+a_{3} \lambda^{3}+a_{2} \lambda^{2}+a_{1} \lambda+a_{0},
$$

where

$$
\begin{aligned}
a_{3} & =D_{x} A_{x}+D_{y} A_{y} \\
a_{2} & =D_{x} D_{y} A_{x} A_{y}+x_{1} y_{1}+x_{2} y_{2} \\
a_{1} & =\left(D_{x} \rho_{x}+D_{y} \rho_{y}\right) x_{1} y_{1}+\left(D_{x} \rho_{x}^{-1}+D_{y} \rho_{y}^{-1}\right) x_{2} y_{2} \\
a_{0} & =D_{x} D_{y}\left(x_{1} \rho_{y}+x_{2} \rho_{x}^{-1}\right)\left(y_{1} \rho_{x}+y_{2} \rho_{y}^{-1}\right)+x_{1} x_{2} y_{1} y_{2} \\
A_{x} & :=\rho_{x}+\rho_{x}^{-1} \\
A_{y} & :=\rho_{y}+\rho_{y}^{-1}
\end{aligned}
$$

All coefficients of $p(\lambda)$ are clearly positive. Furthermore,

$a_{2} a_{3}-a_{1}=\left(D_{x} A_{x}+D_{y} A_{y}\right) A_{x} A_{y}+\left(D_{x} \rho_{x}+D_{y} \rho_{y}\right) x_{2} y_{2}+\left(D_{x} \rho_{x}^{-1}+D_{y} \rho_{y}^{-1}\right) x_{1} y_{1}>0$. 
The key quantity, determining the stability of the positive equilibrium, is $\mathcal{D}=$ $a_{1}\left(a_{2} a_{3}-a_{1}\right)-a_{0} a_{3}^{2}$, which after tedious calculations can be expressed as

$$
\begin{aligned}
\mathcal{D} & =\left(D_{x}^{2}+D_{x} D_{y}\left(\rho_{x} \rho_{y}^{-1}+\rho_{y} \rho_{x}^{-1}\right)+D_{y}^{2}\right)\left(x_{1} y_{1}-x_{2} y_{2}\right)^{2} \\
& +D_{x}^{2} D_{y}\left(D_{x} A_{x}+D_{y} A_{y}\right) x_{2} y_{2}\left(\rho_{x}-1\right)\left(\rho_{x}^{2}+1\right)\left(1-\rho_{x}^{-2} \rho_{y}\right) \\
& +D_{x} D_{y}^{2}\left(D_{x} A_{x}+D_{y} A_{y}\right) x_{2} y_{2}\left(\rho_{y}-1\right)\left(\rho_{y}^{2}+1\right)\left(1-\rho_{y}^{-2} \rho_{x}\right) .
\end{aligned}
$$

The term on the first line is always nonnegative. The terms on the second and third lines are strictly positive provided that at the positive equilibrium the inequality $D_{y} \leq D_{y}^{\text {crit }}$, or equivalently, $\rho_{x} \leq 1<\rho_{y}$ holds. Thus, $\mathcal{D}>0$, and $E_{x y}^{*}$ is locally asymptotically stable by the Routh-Hurwitz criterion in this case. We were unable to show that $\mathcal{D}>0$ even if $D_{y}>D_{y}^{\text {crit }}$, although we believe it to be the case.

\section{C.4 Sensitivity of the steady-state ratios to the diffusivities}

Theorem 5. Suppose that $D_{x}>0$ and $D_{y}>0$, and let $\rho_{x}$ and $\rho_{y}$ be the prey and predator steady-state ratios from Theorem 4. If $\rho_{x}<1$, or equivalently, if $D_{y}<D_{y}^{\text {crit }}$, then

$$
\frac{\partial \rho_{x}}{\partial D_{x}}>0, \quad \frac{\partial \rho_{y}}{\partial D_{x}}>0, \quad \frac{\partial \rho_{x}}{\partial D_{y}}>0, \quad \frac{\partial \rho_{y}}{\partial D_{y}}<0 .
$$

If $\rho_{x}>1$, or equivalently, if $D_{y}>D_{y}^{\text {crit }}$, then

$$
\frac{\partial \rho_{x}}{\partial D_{x}}<0, \quad \frac{\partial \rho_{y}}{\partial D_{x}}<0, \quad \frac{\partial \rho_{x}}{\partial D_{y}}>0, \quad \frac{\partial \rho_{y}}{\partial D_{y}}<0
$$

Proof. The proof of Theorem 4 revealed that the positive steady state $E_{x y}^{*}$ can be expressed in terms of the ratios $\rho_{x}=x_{1} / x_{2}$ and $\rho_{y}=y_{1} / y_{2}$, see (30)-(33). Consequently, we can study the changes in these ratios as the diffusion rates $D_{x}$ and $D_{y}$ are varied. Specifically, implicit differentiation with respect to $D_{x}$ results in the following system

$$
\frac{\partial \rho_{x}}{\partial D_{x}}-\frac{\partial F}{\partial \rho_{y}} \frac{\partial \rho_{y}}{\partial D_{x}}=0, \quad \frac{\partial \rho_{y}}{\partial D_{x}}-\frac{\partial G}{\partial \rho_{x}} \frac{\partial \rho_{x}}{\partial D_{x}}=\frac{\partial G}{\partial D_{x}},
$$

which implies that

$$
\frac{\partial \rho_{x}}{\partial D_{x}}=\frac{\frac{\partial F}{\partial \rho_{y}} \frac{\partial G}{\partial D_{x}}}{1-\frac{\partial F}{\partial \rho_{y}} \frac{\partial G}{\partial \rho_{x}}}, \quad \frac{\partial \rho_{y}}{\partial D_{x}}=\frac{\frac{\partial G}{\partial D_{x}}}{1-\frac{\partial F}{\partial \rho_{y}} \frac{\partial G}{\partial \rho_{x}}} .
$$

Similarly, implicit differentiation with respect to $D_{y}$ results in the following system

$$
\frac{\partial \rho_{x}}{\partial D_{y}}-\frac{\partial F}{\partial \rho_{y}} \frac{\partial \rho_{y}}{\partial D_{y}}=\frac{\partial F}{\partial D_{y}}, \quad \frac{\partial \rho_{y}}{\partial D_{y}}-\frac{\partial G}{\partial \rho_{x}} \frac{\partial \rho_{x}}{\partial D_{y}}=0,
$$

which implies that

$$
\frac{\partial \rho_{x}}{\partial D_{y}}=\frac{\frac{\partial F}{\partial D_{y}}}{1-\frac{\partial F}{\partial \rho_{y}} \frac{\partial G}{\partial \rho_{x}}}, \quad \frac{\partial \rho_{y}}{\partial D_{x}}=\frac{\frac{\partial F}{\partial D_{y}} \frac{\partial G}{\partial \rho_{x}}}{1-\frac{\partial F}{\partial \rho_{y}} \frac{\partial G}{\partial \rho_{x}}} .
$$


The specific functional forms of $F$ and $G$ imply that

$$
\operatorname{sign}\left(\frac{\partial F}{\partial D_{y}}\right)=\operatorname{sign}\left(\rho_{y}-1\right), \quad \operatorname{sign}\left(\frac{\partial G}{\partial D_{x}}\right)=\operatorname{sign}\left(1-\rho_{x}\right),
$$

and we have previously shown that

$$
\frac{\partial F}{\partial \rho_{y}}>0, \quad \frac{\partial G}{\partial \rho_{x}}<0
$$

Therefore, in the case $D_{y}<D_{y}^{c r i t}\left(\rho_{x}<1<\rho_{y}\right)$, we have that

$$
\frac{\partial \rho_{x}}{\partial D_{x}}>0, \quad \frac{\partial \rho_{y}}{\partial D_{x}}>0, \quad \frac{\partial \rho_{x}}{\partial D_{y}}>0, \quad \frac{\partial \rho_{y}}{\partial D_{y}}<0 .
$$

In the case $D_{y}>D_{y}^{\text {crit }}\left(1<\rho_{x}, \rho_{y}\right)$, we have that

$$
\frac{\partial \rho_{x}}{\partial D_{x}}<0, \quad \frac{\partial \rho_{y}}{\partial D_{x}}<0, \quad \frac{\partial \rho_{x}}{\partial D_{y}}>0, \quad \frac{\partial \rho_{y}}{\partial D_{y}}<0 .
$$

We conclude that if the predator diffusivity is sufficiently small, then increasing both diffusion rates has a positive effect on the prey ratios, whereas the predator ratios increase with increasing $D_{x}$ but decrease with increasing $D_{y}$. If the predator diffusivity is large, then the trends changes, namely: increasing both diffusivities has a negative effect on the predator ratios, while the prey ratios increase with increasing $D_{y}$ but decrease with increasing $D_{x}$.

\section{C.5 Other equilibria}

Recall the 2-patch predator-prey model.

$$
\begin{aligned}
& \dot{x}_{1}=x_{1}\left(r-y_{1}\right)+D_{x}\left(x_{2}-x_{1}\right) \\
& \dot{y}_{1}=y_{1}\left(x_{1}-d\right)+D_{y}\left(y_{2}-y_{1}\right) \\
& \dot{x}_{2}=x_{2}\left(\tilde{r}-y_{2}\right)+D_{x}\left(x_{1}-x_{2}\right) \\
& \dot{y}_{2}=y_{2}\left(x_{2}-\tilde{d}\right)+D_{y}\left(y_{1}-y_{2}\right)
\end{aligned}
$$

We have previously focused on the existence and stability of positive steady states, and here we consider boundary steady states. We have that

Theorem 6. $E_{0}^{*}=(0,0,0,0)$ is always an unstable steady state of system (38).

When $D_{x}=0$, then in addition to the positive steady state $E_{y}^{*}$, which exists for all $D_{y}<\tilde{r} \tilde{d} /(r-\tilde{r})$, there are boundary steady states

$$
E_{\mathrm{FG}}^{*}=\left(0, \frac{\tilde{r} D_{y}}{d+D_{y}}, \frac{\left(d+D_{y}\right)\left(\tilde{d}+D_{y}\right)-D_{y}^{2}}{d+D_{y}}, \tilde{r}\right)
$$

and

$$
E_{\mathrm{MPA}}^{*}=\left(\frac{\left(d+D_{y}\right)\left(\tilde{d}+D_{y}\right)-D_{y}^{2}}{\tilde{d}+D_{y}}, r, 0, \frac{r D_{y}}{\tilde{d}+D_{y}}\right)
$$


which exist for all $D_{y} . E_{F G}^{*}$ is always unstable, whereas $E_{M P A}^{*}$ is unstable if $D_{y}<$ $\tilde{r} \tilde{d} /(r-\tilde{r})$, but globally stable with respect to positive solutions if $D_{y}>\tilde{r} \tilde{d} /(r-\tilde{r})$.

When $D_{y}=0$, then in addition to the positive steady state $E_{x}^{*}$, which exists for all $D_{x}<\tilde{r} \tilde{d} /(\tilde{d}-d)$, there are boundary steady states

$$
E_{\mathrm{FG}}^{* *}=\left(\frac{\tilde{d} D_{x}}{D_{x}-r}, 0, \tilde{d}, \tilde{r}+\frac{r D_{x}}{D_{x}-r}\right),
$$

which exists if and only if $D_{x}>r$, and

$$
E_{\mathrm{MPA}}^{* *}=\left(d, r+\frac{\tilde{r} D_{x}}{D_{x}-\tilde{r}}, \frac{d D_{x}}{D_{x}-\tilde{r}}, 0\right),
$$

which exists if and only if $D_{x}>\tilde{r} . E_{\mathrm{FG}}^{* *}$ is unstable whenever it exists, and $E_{\mathrm{MPA}}^{* *}$ is unstable if $\tilde{r}<D_{x}<\tilde{r} \tilde{d} /(\tilde{d}-d)$, but globally stable with respect to positive solutions if $D_{x}>\tilde{r} \tilde{d} /(\tilde{d}-d)$.

Proof. System (38) has Jacobian matrix:

$$
J=\left[\begin{array}{cccc}
r-y_{1}-D_{x} & -x_{1} & D_{x} & 0 \\
y_{1} & x_{1}-d-D_{y} & 0 & D_{y} \\
D_{x} & 0 & \tilde{r}-y_{2}-D_{x} & -x_{2} \\
0 & D_{y} & y_{2} & x_{2}-\tilde{d}-D_{y}
\end{array}\right]
$$

Clearly,

$$
E_{0}^{*}=(0,0,0,0)
$$

is always an equilibrium and the Jacobian at $E_{0}^{*}$ has the form

$$
\left[\begin{array}{cccc}
r-D_{x} & 0 & D_{x} & 0 \\
0 & -d-D_{y} & 0 & D_{y} \\
D_{x} & 0 & \tilde{r}-D_{x} & 0 \\
0 & D_{y} & 0 & -\tilde{d}-D_{y}
\end{array}\right]
$$

and after rearranging the matrix, we find that two of its eigenvalues are the eigenvalues of the submatrix

$$
\left[\begin{array}{cc}
r-D_{x} & D_{x} \\
D_{x} & \tilde{r}-D_{x}
\end{array}\right]
$$

This submatrix has a positive eigenvalue whenever $r+\tilde{r}>0$. Thus, $E_{0}^{*}$ is always unstable.

Case: $D_{x}=0$.

It is easily verified that $E_{\mathrm{FG}}^{*}$ and $E_{\mathrm{MPA}}^{*}$ are boundary steady states which exist for all $D_{y}$. The Jacobian at $E_{\mathrm{FG}}^{*}$ has an eigenvalue

$$
r-\frac{D_{y} \tilde{r}}{d+D_{y}}>r-\tilde{r} \geq 0,
$$

so $E_{\mathrm{FG}}^{*}$ is always unstable. The Jacobian at $E_{\mathrm{MPA}}^{*}$ is

$$
J\left(E_{\mathrm{MPA}}^{*}\right)=\left[\begin{array}{cccc}
0 & -\frac{\left(d+D_{y}\right)\left(\tilde{d}+D_{y}\right)-D_{y}^{2}}{\tilde{d}+D_{y}} & 0 & 0 \\
r & \frac{\left(d+D_{y}\right)\left(\tilde{d}+D_{y}\right)-D_{y}^{2}}{\tilde{d}+D_{y}}-d-D_{y} & 0 & D_{y} \\
0 & 0 & \tilde{r}-\frac{r D_{y}}{\tilde{d}+D_{y}} & 0 \\
0 & D_{y} & \frac{r D_{y}}{\tilde{d}+D_{y}} & -\tilde{d}-D_{y}
\end{array}\right] .
$$


The eigenvalues are

$$
\lambda_{1}=\tilde{r}-\frac{r D_{y}}{\tilde{d}+D_{y}}=\frac{\tilde{r} \tilde{d}-f_{x} D_{y}}{\tilde{d}+D_{y}}
$$

and the eigenvalues of

$$
\left[\begin{array}{ccc}
0 & -\frac{\left(d+D_{y}\right)\left(\tilde{d}+D_{y}\right)-D_{y}^{2}}{\tilde{d}+D_{y}} & 0 \\
r & \frac{\left(d+D_{y}\right)\left(\tilde{d}+D_{y}\right)-D_{y}^{2}}{\tilde{d}+D_{y}}-d-D_{y} & D_{y} \\
0 & D_{y} & -\tilde{d}-D_{y}
\end{array}\right],
$$

which has characteristic polynomial

$$
\begin{aligned}
\lambda^{3}+a_{1} \lambda^{2}+a_{2} \lambda+a_{3} & =0, \\
a_{1} & =\frac{\left(\tilde{d}+D_{y}\right)^{2}+D_{y}^{2}}{\tilde{d}+D_{y}}, \\
a_{2} & =r \frac{\left(d+D_{y}\right)\left(\tilde{d}+D_{y}\right)-D_{y}^{2}}{\tilde{d}+D_{y}}, \\
a_{3} & =r\left[\left(d+D_{y}\right)\left(\tilde{d}+D_{y}\right)-D_{y}^{2}\right] .
\end{aligned}
$$

The Routh-Hurwitz stability conditions are satisfied: $a_{1}>0, a_{3}>0$, and

$$
a_{1} a_{2}-a_{3}=\frac{r D_{y}^{2}}{\left(\tilde{d}+D_{y}\right)^{2}}\left[\left(d+D_{y}\right)\left(\tilde{d}+D_{y}\right)-D_{y}^{2}\right]>0
$$

so these three eigenvalues have negative real parts. Therefore, the stability of $E_{\mathrm{MPA}}^{*}$ is determined exclusively by $\lambda_{1}$ : for $D_{y}<\frac{\tilde{r} \tilde{d}}{r-\tilde{r}}, E_{\mathrm{MPA}}^{*}$ is unstable and for $D_{y}>\frac{\tilde{r} \tilde{d}}{r-\tilde{r}}$, it is stable. Recall that the positive steady state $E_{y}^{*}$ exists so long as $D_{y}<\frac{\tilde{r} \tilde{d}}{r-\tilde{r}}$, and that it is globally stable. When $D_{y}=\frac{\tilde{r} \tilde{d}}{r-\tilde{r}}, E_{y}^{*}$ ceases to exist as a positive steady state because it collides with $E_{\mathrm{MPA}}^{*}$, which becomes locally stable when $D_{y}>\frac{\tilde{r} \tilde{d}}{r-\tilde{r}}$. Thus, there appears to be a transcritical bifurcation at $D_{y}=\frac{\tilde{r} \tilde{d}}{r-\tilde{r}}$.

As before, if $D_{y}>\frac{\tilde{r} \tilde{d}}{r-\tilde{r}}$, we let

$$
\phi_{y}^{0}:=\frac{y_{1}^{*}}{y_{2}^{*}}=\frac{D_{y}+\tilde{d}}{D_{y}},
$$

and use the familiar Lyapunov function to establish that $E_{\mathrm{MPA}}^{*}$ is globally stable with respect to positive solutions:

$$
\begin{aligned}
V\left(x_{1}, y_{1}, x_{2}, y_{2}\right)= & \int_{x_{1}^{*}}^{x_{1}}\left(1-\frac{x_{1}^{*}}{s}\right) d s+\int_{y_{1}^{*}}^{y_{1}}\left(1-\frac{y_{1}^{*}}{s}\right) d s \\
& +\left(\phi_{y}^{0}\right)^{-1}\left(x_{2}+\int_{y_{2}^{*}}^{y_{2}}\left(1-\frac{y_{2}^{*}}{s}\right) d s\right),
\end{aligned}
$$

where now $\left(x_{1}^{*}, y_{1}^{*}, x_{2}^{*}, y_{2}^{*}\right)=E_{\mathrm{MPA}}^{*}$. After some algebraic manipulations, we find that

$$
\dot{V}=-D_{y} \frac{y_{1}}{y_{2}} y_{1}^{*}\left(\frac{y_{2}}{y_{1}}-\frac{1}{\phi_{y}^{0}}\right)^{2}+\frac{x_{2}}{\phi_{y}^{0}}\left(\tilde{r}-y_{2}^{*}\right),
$$


which is clearly nonpositive since $\tilde{r}-y_{2}^{*}<0$ due to the stability condition $D_{y}>\frac{\tilde{r} \tilde{d}}{r-\tilde{r}}$. We also have that $\dot{V}=0$ if and only if $\frac{y_{1}}{y_{2}}=\phi_{y}^{0}$ and $x_{2}=0$. Consider a solution that satisfies $\dot{V}=0$ for all $t$. We have that

$$
\frac{\dot{y}_{2}(t)}{y_{2}(t)} \equiv-\tilde{d}+D_{y}\left(\phi_{y}^{0}-1\right)=0
$$

hence $y_{2}(t)$ must be constant. Consequently, $y_{1}(t)$ must also be constant. Therefore,

$$
0 \equiv \frac{\dot{y}_{1}(t)}{y_{1}(t)}=x_{1}(t)-d+D_{y}\left(\frac{1}{\phi_{y}^{0}}-1\right)=x_{1}(t)-x_{1}^{*},
$$

implying that $x_{1}(t) \equiv x_{1}^{*}$. This, in turn, implies that $y_{1}(t) \equiv y_{1}^{*}$ and $y_{2}(t) \equiv y_{2}^{*}$. Thus the only invariant set contained in the set where $\dot{V}=0$, is $\left\{E_{\mathrm{MPA}}^{*}\right\}$ and this equilibrium attracts all positive solutions.

Case: $D_{y}=0$. It is easily verified that $E_{\mathrm{FG}}^{* *}$ and $E_{\mathrm{MPA}}^{* *}$ are boundary steady states which exist for the values of $D_{x}$ indicated in the Theorem. The Jacobian matrix at $E_{\mathrm{FG}}^{* *}$ has an eigenvalue $\lambda=\frac{\tilde{d} D_{x}}{D_{x}-r}-d>\tilde{d}-d \geq 0$, hence $E_{\mathrm{FG}}^{* *}$ is unstable whenever it exists. The local stability of $E_{\mathrm{MPA}}^{* *}$ is determined by the eigenvalues of the corresponding Jacobian matrix

$$
J\left(E_{\mathrm{MPA}}^{* *}\right)=\left[\begin{array}{cccc}
-\alpha & -d & D_{x} & 0 \\
y_{1}^{* *} & 0 & 0 & 0 \\
D_{x} & 0 & -\beta & -x_{2}^{* *} \\
0 & 0 & 0 & x_{2}^{* *}-\tilde{d}
\end{array}\right]
$$

where now $E_{\mathrm{MPA}}^{* *}=\left(x_{1}^{* *}, y_{1}^{* *}, x_{2}^{* *}, y_{2}^{* *}\right)$, and $\alpha=\frac{D_{x}^{2}}{D_{x}-\tilde{r}}>0$, and $\beta=D_{x}-\tilde{r}>0$. Clearly, one of the eigenvalues is equal to

$$
\lambda_{4}:=x_{2}^{* *}-\tilde{d}=\frac{d D_{x}}{D_{x}-\tilde{r}}-\tilde{d}
$$

and it is negative if and only if

$$
D_{x}>\frac{\tilde{r} \tilde{d}}{\tilde{d}-d}>\tilde{r}
$$

The three remaining eigenvalues are also eigenvalues of the submatrix

$$
\left[\begin{array}{ccc}
-\alpha & -d & D_{x} \\
y_{1}^{* *} & 0 & 0 \\
D_{x} & 0 & -\beta
\end{array}\right]
$$

which has the characteristic polynomial of the form

$$
p(z)=z^{3}+(\alpha+\beta) z^{2}+d y_{1}^{* *} \lambda+\beta d y_{1}^{* *},
$$

and therefore is Hurwitz. We conclude that $E_{\mathrm{MPA}}^{* *}$ is locally stable if $D_{x}>\frac{\tilde{r} \tilde{d}}{\tilde{d}-d}$ and unstable if $\frac{\tilde{r} \tilde{d}}{\tilde{d}-d}>D_{x}>\tilde{r}$. 
To show that $D_{x}>\frac{\tilde{r} \tilde{d}}{\tilde{d}-d}$ implies that $E_{\text {MPA }}^{* *}$ attracts all positive solutions, we define $\phi_{x}^{0}:=\frac{x_{1}^{* *}}{x_{2}^{* *}}$, and an auxiliary function

$$
\begin{gathered}
V\left(x_{1}, y_{1}, x_{2}, y_{2}\right)=\int_{x_{1}^{* *}}^{x_{1}}\left(1-\frac{x_{1}^{* *}}{s}\right) d s+\int_{y_{1}^{* *}}^{y_{1}}\left(1-\frac{y_{1}^{* *}}{s}\right) d s \\
+\frac{1}{\phi_{x}^{0}}\left(\int_{x_{2}^{* *}}^{x_{2}}\left(1-\frac{x_{2}^{* *}}{s}\right) d s+y_{2}\right),
\end{gathered}
$$

After some tedious algebra, we find that

$$
\dot{V}=\frac{-x_{1}^{* *} D_{x}\left(\frac{x_{2}}{x_{1}}-\left(\phi_{x}^{0}\right)^{-1}\right)^{2}}{\frac{x_{2}}{x_{1}}}+\frac{y_{2}}{\phi_{x}^{0}}\left(x_{2}^{* *}-\tilde{d}\right) \leq 0,
$$

hence $V$ is a Lyapunov function. An argument similar to those given above and therefore omitted establishes that $E_{\mathrm{MPA}}^{* *}$ attracts all positive solutions. 


\section{References}

[1] Abesamis, R.A., and Russ, G.R., Density-dependent spillover from a marine reserve: long-term evidence, Ecological Applications 15(5), 1798-1812, 2005.

[2] Beverton, R.J.H., and Holt, S.J., On the dynamics of exploited fish populations, Springer Science, 1957.

[3] Barnett, L. A., and Baskett, M. L., Marine reserves can enhance ecological resilience, Ecology Letters 18 (12), 1301-1310, 2015.

[4] Baskett, M.L., Micheli, F., and Levin, S.A., Designing marine reserves for interacting species: Insights from theory, Biological Conservation 137, 163$179,2007$.

[5] Baskett, M. L., and Barnett, L. A., The Ecological and Evolutionary Consequences of Marine Reserves. Annual Review of Ecology, Evolution, and Systematics, 46 (1), 2015.

[6] Bazykin, A.D., Nonlinear Dynamics of Interacting Populations, World Scientific, 1998.

[7] Botsford, L.W., Hastings, A., and Gaines, S.D., Dependence of sustainability on the configuration of marine reserves and larval dispersal distance, Ecology Letters 4, 144-150, 2001.

[8] Cantrell, R.S., and Cosner, C., Models for predator-prey systems at multiple scales, SIAM Review 38 (2), 256-286, 1996.

[9] Cantrell, R.S., and Cosner, C., Spatial Ecology via Reaction-Diffusion Equations, Wiley, 2003.

[10] Claudet, J., Osenberg, C.W., Domenici, P., Badalamenti, F., Milazzo, M., Falcón, J.M., Bertocci, I., Benedetti-Cecchi, L., Garcia-Charton, J.A., Goñi, R., Borg, J.A., Forcada, A., De Lucia, A., Pérez-Ruzafa, A., Afonso, P., Brito, A., Guala, I., Le Diréach, L., Sanchez-Jerez, P., Somerfield, P.J., and Planes, S., Marine reserves: fish life history and ecological traits matter. Ecological Applications 20, 830-839, 2010.

[11] Claudet, J., Osenberg, C. W., Benedetti-Cecchi, L., Domenici, P., GarciaCharton, J.A., Perez-Ruzafa, A., Badalamenti, F., Bayle-Sempere, J., Brito, A., Bulleri, F., Culioli, J.M., Dimech, M., Falcón, J.M., Guala, I., Milazzo, M., Sánchez-Meca, J., Somerfield, P.J., Stobart, B., Vandeperre, F.,Valle, C., and Planes, S., Marine reserves: size and age do matter, Ecology Letters 11,481-489, 2008.

[12] Eggleston, D.B., and Parsons, D.M., Disturbance-induced 'spill-in' of Caribbean lobster to marine reserves. Marine Ecology Progress Series 371, 213-220, 2008. 
[13] Goñi, R., Adlerstein, S., Alvarez-Berastegui, D., Forcada, A., Reñones, O., Criquet, G., Polti, S., Cadiou, G., Valle, C., Lenfant, P., Bonhomme, P., Pérez-Ruzafa, A., Sánchez-Lizaso, F.L., Garcia-Charton, J.A., Bernard, G., Stelzenmüller, V., Planes, S., Spillover from six western Mediterranean marine protected areas: evidence from artisanal fisheries, Marine Ecology Progress Series 366,159-174, 2008.

[14] Hadeler, K.P., Quiescence, excitability, and heterogeneity in ecological models, Journal of Mathematical Biology 66, 649-684, 2013.

[15] Hale, J.K., Diffusive coupling, dissipation, and synchronization, Journal of Dynamics and Differential Equations 9(1), 1-52, 1997.

[16] Halpern, B.S., The impact of marine reserves: do reserves work and does reserve size matter? Ecological Applications 13, 117-137, 2003.

[17] Hastings, A., Global stability in Lotka-Volterra systems with diffusion, Journal of Mathematical Biology 6, 163-168, 1978.

[18] Hastings, A., and Botsford, L.W., Equivalence in yield from marine reserves and traditional fisheries management, Science 284,1537-1538, 1999.

[19] Holland, M.D., and Hastings, A., Strong effects of dispersal network structure on ecological dynamics, Nature 456, 792-795, 2008.

[20] Holt, R.D., Population dynamics in two-patch environments: some anomalous consequences of an optimal habitat distribution, Theoretical Population Biology 28 (2), 181-208, 1985.

[21] Huang, Y., and Diekmann, O., Interspecific Influence on Mobility and Turing Instability, Bulletin of Mathematical Biology 65,143-156, 2003.

[22] Huang, Y., and Diekmann, O., Predator migration in response to prey density: What are the consequences?, Journal of Mathematical Biology 43, 561-581, 2001.

[23] Jansen, V.A.A., Regulation of predator-prey systems through spatial interactions: a possible solution to the Paradox of Enrichment, Oikos 74 (3), 384-390, 1995.

[24] Kellner, J.B., Tetreault, I., Gaines, S.D. and Nisbet, R.M., Fishing the line near marine reserves in single and multispecies fisheries, Ecological Applications 17, 1039-1054, 2007.

[25] Kellner, J. B., and Hastings, A., A reserve paradox: introduced heterogeneity may increase regional invasibility. Conservation Letters, 2(3), 115-122 (2009).

[26] Kellner, J. B., Litvin, S. Y., Hastings, A., Micheli, F., and Mumby, P. J., Disentangling trophic interactions inside a Caribbean marine reserve. Ecological Applications, 20(7), 1979-1992 (2010). 
[27] Kot, M., Elements of Mathematical Ecology, Cambridge University Press, 2001.

[28] Kuang, Y., and Beretta, E., Global qualitative analysis of a ratio-dependent predatorprey system, Journal of Mathematical Biology 36, 389-406, 1998.

[29] Kuang, Y., and Takeuchi, Y., Predator-prey dynamics in models of prey dispersal in two-patch environments, Mathematical Biosciences 120, 77-98, 1994.

[30] Langebrake, J., Riotte-Lambert, L., Osenberg, C.W., and De Leenheer, P., Differential movement and movement bias models for marine protected areas, Journal of Mathematical Biology 64, 667-696, 2012.

[31] Liu, Y., The dynamical behavior of a two patch predator-prey model, Bachelors Thesis, The College of William and Mary, 2010.

[32] Lotka, A.J., The growth of mixed populations, two species competing for a common food supply, Journal of the Washington Academy of Sciences 22, 461-469, 1932.

[33] Maciel, G.A., and Kraenkel, R.A., How population loss through habitat boundaries determines the dynamics of a predator-prey system, Ecological Complexity 20, 33-42, 2014.

[34] Mangel, M., No-take areas for sustainability of harvested species and a conservation invariant for marine reserves, Ecology Letters 1, 97-90, 1998.

[35] Micheli, F., Amarasekare, P., Bascompte, J., and Gerber, L. R., Including species interactions in the design and evaluation of marine reserves: some insights from a predator-prey model. Bulletin of Marine Science, 74(3), 653669, 2004.

[36] Moffitt, E.A., Botsford, L.W. , Kaplan, D.M., and O'Farrell, M.R., Marine reserve networks for species that move within a home range, Ecological Applications 19(7), 1835-1847, 2009.

[37] Osenberg, C.W., Shima, J.S., Miller, S.L. , and Stier, A.C. . Assessing effects of marine protected areas: confounding in space and possible solutions. p. 143167. In J. Claudet (ed) Marine Protected Areas: A multidisciplinary approach, Cambridge University Press, 2011.

[38] Pérez-Ruzafa, A., etal, Modeling spatial and temporal scales for spill-over and biomass exportation from MPAs and their potential for fisheries enhancement, Journal for Nature Conservation 16, 234-255, 2008.

[39] Polacheck, T., Year around closed areas as a management tool, Natural Resource Modeling 4, 327-354, 1990.

[40] Roberts, C.M., Bohnsack, J. A., Gell, F.,Hawkins, J.P., and Goodridge, R., Effects of marine reserves on adjacent fisheries, Science 294, 1920-1923, 2001. 
[41] de Roos, A.M., and Persson, L., Population and Community Ecology of Ontogenetic Development, Princeton University Press, 2013.

[42] Rosenzweig, M.L., and MacArthur, R., Graphical representation and stability conditions of predator-prey interactions. American Naturalist 97, 209-223, 1963.

[43] Sale, P.F., etal, Critical science gaps impede use of no-take fishery reserves. Trends in Ecology and Evolution 20, 74-80, 2005.

[44] Smith, H.L., Monotone Dynamical Systems, American Mathematical Society, 1995.

[45] Takashina, N., Mougi, A., and Iwasa, Y., Paradox of marine protected areas: suppression of fishing may cause species loss. Population ecology, 54(3), 475485 (2012).

[46] Volterra, V., Fluctuation in the abundance of a species considered mathematically, Nature 118, 558-560, 1926.

[47] Walters, C., Pauly, D., and Christensen, V., Ecospace: Prediction of Mesoscale Spatial Patterns in Trophic Relationships of Exploited Ecosystems, with Emphasis on the Impacts of Marine Protected Areas, Ecosystems 2, 539-554, 1999.

[48] Walters, C., Impacts of dispersal, ecological interactions, and fishing effort dynamics on efficacy of marine protected areas: How large should protected areas be?, Bulletin of Marine Science 66, 754-757, 2000.

[49] White, J.W., Spatially Coupled Larval Supply of Marine Predators and Their Prey Alters the Predictions of Metapopulation Models, The American Naturalist 171, E179-E194, 2008.

[50] White, J.W., and Samhouri, J.F., Oceanographic coupling across three trophic levels shapes sourcesink dynamics in marine metacommunities, Oikos 120, 1151-1164, 2011.

[51] White, J. W., Botsford, L. W., Baskett, M. L., Barnett, L. A., Barr, R. J., and Hastings, A., Linking models with monitoring data for assessing performance of no-take marine reserves. Frontiers in Ecology and the Environment, 9(7), 390-399, 2011. 